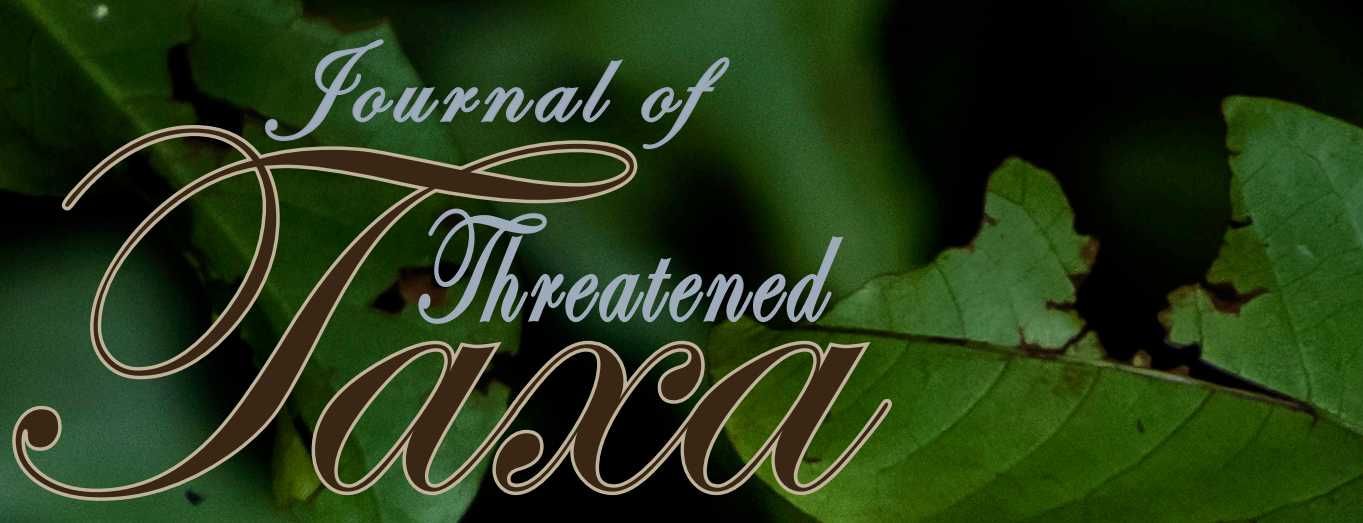

Building exidence for conservation glabally

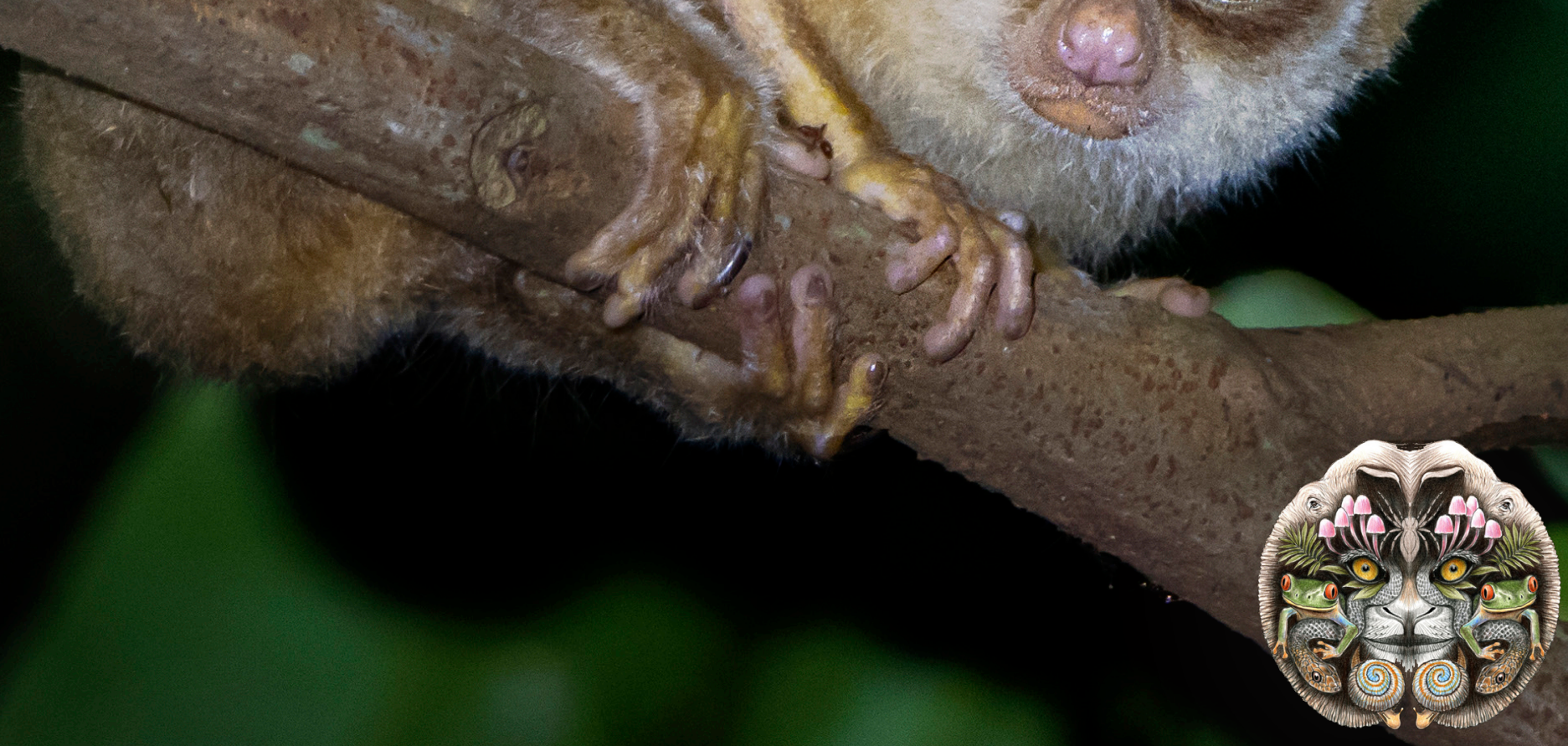

Open Access

$10.11609 /$ jott.2021.13.010.19431-19614 creven.threatenedtaxa.arg

26 September 2021 (Online \& Print) Val. 13 | Na. 11 | Pages: 19431-19674 


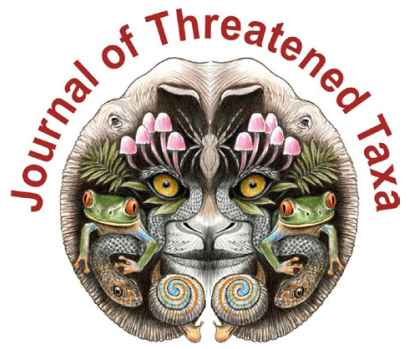

ISSN 0974-7907 (Online); ISSN $0974-7893$ (Print)

Publisher

Host

Wildlife Information Liaison Development Society

www.wild.zooreach.org

Zoo Outreach Organization www.zooreach.org

No. 12, Thiruvannamalai Nagar, Saravanampatti - Kalapatti Road, Saravanampatti, Coimbatore, Tamil Nadu 641035, India

Ph: +91 9385339863 | www.threatenedtaxa.org

Email: sanjay@threatenedtaxa.org

EDITORS

\section{Founder \& Chief Editor}

Dr. Sanjay Molur

Wildlife Information Liaison Development (WILD) Society \& Zoo Outreach Organization (ZOO)

12 Thiruvannamalai Nagar, Saravanampatti, Coimbatore, Tamil Nadu 641035, India

\section{Deputy Chief Edito}

Dr. Neelesh Dahanukar

Noida, Uttar Pradesh, India

\section{Managing Editor}

Mr. B. Ravichandran, WILD/ZOO, Coimbatore, India

\section{Associate Editors}

Dr. Mandar Paingankar, Government Science College Gadchiroli, Maharashtra 442605, India

Dr. Ulrike Streicher, Wildlife Veterinarian, Eugene, Oregon, USA

Ms. Priyanka Iyer, ZOO/WILD, Coimbatore, Tamil Nadu 641035, India

Dr. B. A. Daniel, $200 / \mathrm{WILD}$, Coimbatore, Tamil Nadu 641035, India

\section{Editorial Board}

Dr. Russel Mittermeier

Executive Vice Chair, Conservation International, Arlington, Virginia 22202, USA

\section{Prof. Mewa Singh Ph.D., FASc, FNA, FNASc, FNAPsy}

Ramanna Fellow and Life-Long Distinguished Professor, Biopsychology Laboratory, and Institute of Excellence, University of Mysore, Mysuru, Karnataka 570006, India; Honorary Professor, Jawaharlal Nehru Centre for Advanced Scientific Research, Bangalore; and Adjunct Professor, National Institute of Advanced Studies, Bangalore

\section{Stephen D. Nash}

Scientific Illustrator, Conservation International, Dept. of Anatomical Sciences, Health Sciences Center, T-8, Room 045, Stony Brook University, Stony Brook, NY 11794-8081, USA

\section{Dr. Fred Pluthero}

Toronto, Canada

\section{Dr. Priya Davidar}

Sigur Nature Trust, Chadapatti, Mavinhalla PO, Nilgiris, Tamil Nadu 643223, India

\section{Dr. Martin Fisher}

Senior Associate Professor, Battcock Centre for Experimental Astrophysics, Cavendish

Laboratory, JJ Thomson Avenue, Cambridge CB3 OHE, UK

\section{Dr. John Fellowe}

Honorary Assistant Professor, The Kadoorie Institute, 8/F, T.T. Tsui Building, The University of Hong Kong, Pokfulam Road, Hong Kong

\section{Prof. Dr. Mirco Solé}

Universidade Estadual de Santa Cruz, Departamento de Ciências Biológicas, Vice-coordenado do Programa de Pós-Graduação em Zoologia, Rodovia Ilhéus/Itabuna, Km 16 (45662-000)

Salobrinho, Ilhéus - Bahia - Brasil

\section{Dr. Rajeev Raghavan}

Professor of Taxonomy, Kerala University of Fisheries \& Ocean Studies, Kochi, Kerala, India

\section{English Editors}

Mrs. Mira Bhojwani, Pune, India

Dr. Fred Pluthero, Toronto, Canad

Mr. P. Ilangovan, Chennai, India

Web Maintenance

Mrs. Latha G. Ravikumar, ZOO/WILD, Coimbatore, India

\section{Typesetting}

Mr. Arul Jagadish, ZOO, Coimbatore, India

Mrs. Radhika, ZOO, Coimbatore, India

Mrs. Geetha, ZOO, Coimbatore India

\section{Fundraising/Communications}

Mrs. Payal B. Molur, Coimbatore, India

Subject Editors 2018-2020

Fungi

Dr. B. Shivaraju, Bengaluru, Karnataka, India

Dr. R.K. Verma, Tropical Forest Research Institute, Jabalpur, India

Dr. Vatsavaya S. Raju, Kakatiay University, Warangal, Andhra Pradesh, India

Dr. M. Krishnappa, Jnana Sahyadri, Kuvempu University, Shimoga, Karnataka, India

Dr. K.R. Sridhar, Mangalore University, Mangalagangotri, Mangalore, Karnataka, India

Dr. Gunjan Biswas, Vidyasagar University, Midnapore, West Bengal, India

Plants

Dr. G.P. Sinha, Botanical Survey of India, Allahabad, India

Dr. N.P. Balakrishnan, Ret. Joint Director, BSI, Coimbatore, India

Dr. Shonil Bhagwat, Open University and University of Oxford, UK

Prof. D.J. Bhat, Retd. Professor, Goa University, Goa, India

Dr. Ferdinando Boero, Università del Salento, Lecce, Italy

Dr. Dale R. Calder, Royal Ontaro Museum, Toronto, Ontario, Canada

Dr. Cleofas Cervancia, Univ. of Philippines Los Baños College Laguna, Philippines

Dr. F.B. Vincent Florens, University of Mauritius, Mauritius

Dr. Merlin Franco, Curtin University, Malaysia

Dr. V. Irudayaraj, St. Xavier's College, Palayamkottai, Tamil Nadu, India

Dr. B.S. Kholia, Botanical Survey of India, Gangtok, Sikkim, India

Dr. Pankaj Kumar, Kadoorie Farm and Botanic Garden Corporation, Hong Kong S.A.R., China

Dr. V. Sampath Kumar, Botanical Survey of India, Howrah, West Bengal, India

Dr. A.J. Solomon Raju, Andhra University, Visakhapatnam, India

Dr. Vijayasankar Raman, University of Mississippi, USA

Dr. B. Ravi Prasad Rao, Sri Krishnadevaraya University, Anantpur, India

Dr. K. Ravikumar, FRLHT, Bengaluru, Karnataka, India

Dr. Aparna Watve, Pune, Maharashtra, India

Dr. Qiang Liu, Xishuangbanna Tropical Botanical Garden, Yunnan, China

Dr. Noor Azhar Mohamed Shazili, Universiti Malaysia Terengganu, Kuala Terengganu, Malaysia Dr. M.K. Vasudeva Rao, Shiv Ranjani Housing Society, Pune, Maharashtra, India

Prof. A.J. Solomon Raju, Andhra University, Visakhapatnam, India

Dr. Mandar Datar, Agharkar Research Institute, Pune, Maharashtra, India

Dr. M.K. Janarthanam, Goa University, Goa, India

Dr. K. Karthigeyan, Botanical Survey of India, India

Dr. Errol Vela, University of Montpellier, Montpellier, France

Dr. P. Lakshminarasimhan, Botanical Survey of India, Howrah, India

Dr. Larry R. Noblick, Montgomery Botanical Center, Miami, USA

Dr. K. Haridasan, Pallavur, Palakkad District, Kerala, India

Dr. Analinda Manila-Fajard, University of the Philippines Los Banos, Laguna, Philippines

Dr. P.A. Sinu, Central University of Kerala, Kasaragod, Kerala, India

Dr. Afroz Alam, Banasthali Vidyapith (accredited A grade by NAAC), Rajasthan, India

Dr. K.P. Rajesh, Zamorin's Guruvayurappan College, GA College PO, Kozhikode, Kerala, India

Dr. David E. Boufford, Harvard University Herbaria, Cambridge, MA 02138-2020, USA

Dr. Ritesh Kumar Choudhary, Agharkar Research Institute, Pune, Maharashtra, India

Dr. Navendu Page, Wildlife Institute of India, Chandrabani, Dehradun, Uttarakhand, India

Invertebrates

Dr. R.K. Avasthi, Rohtak University, Haryana, India

Dr. D.B. Bastawade, Maharashtra, India

Dr. Partha Pratim Bhattacharjee, Tripura University, Suryamaninagar, India

Dr. Kailash Chandra, Zoological Survey of India, Jabalpur, Madhya Pradesh, India

Dr. Ansie Dippenaar-Schoeman, University of Pretoria, Queenswood, South Africa

Dr. Rory Dow, National Museum of natural History Naturalis, The Netherlands

Dr. Brian Fisher, California Academy of Sciences, USA

Dr. Richard Gallon, llandudno, North Wales, LL30 1UP

Dr. Hemant V. Ghate, Modern College, Pune, India

Dr. M. Monwar Hossain, Jahangirnagar University, Dhaka, Bangladesh

Mr. Jatishwor Singh Irungbam, Biology Centre CAS, Branišovská, Czech Republic

Dr. lan J. Kitching Natural History Museum, Cromwell Road, UK

Dr. George Mathew, Kerala Forest Research Institute, Peechi, India

Dr. John Noyes, Natural History Museum, London, UK

For Focus, Scope, Aims, and Policies, visit https://threatenedtaxa.org/index.php/JoTT/aims_scope
For Article Submission Guidelines, visit https://threatenedtaxa.org/index.php/JoTT/about/submissions
For Policies against Scientific Misconduct, visit https://threatenedtaxa.org/index.php/JoTT/policies_various 


\title{
Birds of Barandabhar Corridor Forest, Chitwan, Nepal
}

\author{
Saneer Lamichhane ${ }^{1} \mathbb{D}$, Babu Ram Lamichhane ${ }^{2}$ (D) Kapil Pokharel $^{3}$ D, Pramod Raj Regmi $^{4}$ (D), \\ Tulasi Prasad Dahal ${ }^{5}$ (D), Santosh Bhattarai ${ }^{6}(\mathbb{D})$, Chiranjibi Prasad Pokheral ${ }^{7}$ (D) Pabitra Gotame ${ }^{8}$ (D), \\ Trishna Rayamajhi ${ }^{9}$ (D) Ram Chandra Kandel ${ }^{10}$ (D) \& Aashish Gurung ${ }^{11}$ (D)
}

\author{
${ }^{1-7,11}$ National Trust for Nature Conservation, Nepal. \\ ${ }^{9}$ Department of Natural Resources, Cornell University, USA. \\ ${ }^{8,10}$ Ministry of Forest and Environment, Government of Nepal. \\ ${ }^{1}$ saneerlamichhane@gmail.com (corresponding author), ${ }^{2}$ baburaml@gmail.com, ${ }^{3}$ ruff kapil@hotmail.com, \\ ${ }^{4}$ regmiprr11@gmail.com, ${ }^{5}$ envoytulasi@gmail.com, ${ }^{6}$ bhattarai.bcc@gmail.com, ${ }^{7}$ pokheralchiran2017@gmail.com, \\ ${ }^{8}$ pabitragotame@gmail.com, ${ }^{9}$ trishnarayamajhi07@gmail.com, ${ }^{10}$ rckandel06@gmail.com, ${ }^{11}$ asis_grg@hotmail.com
}

\begin{abstract}
Long term monitoring of bird species was conducted in Barandabhar Corridor Forest, one of the important bird areas of Nepal (IBA). Bird species were identified by the point count method in transect surveys in two-time frames from 2002-2012 and 2015-2016 to obtain the bird species list. We compared our bird list with previously published (after 2000) checklists and compiled the updated checklist of birds of Barandabhar Corridor Forest. We documented 372 bird species belonging to 80 families in Barandabhar, including five Critically Endangered, three Endangered, eight Vulnerable, and 15 Near Threatened species. The Accipitridae family included the highest number of species $(n=32)$, followed by Muscicapidae $(n=30)$ and Anatidae $(n=18)$. Approximately, half of the total confirmed bird species were insectivorous. The list included $63 \%$ resident, $27 \%$ winter migratory, $7.5 \%$ summer migratory, and $2.9 \%$ passage migrant species. According to the habitat type, there were 181 species of forest, 74 species of wetland, 24 species of grassland, 70 species of open field, and 23 species of partially wetland birds. This updated checklist of bird species will serve as a reference guide for bird watchers, biodiversity researchers, and support managers for conservation effort; and can be used to track any changes in the composition of bird species in the future.
\end{abstract}

Keywords: Avifauna, checklist, IBA, transect survey, wetland birds.

Editor: Carol Inskipp, Bishop Auckland Co., Durham, UK.

Date of publication: 26 September 2021 (online \& print)

Citation: Lamichhane, S., B.R. Lamichhane, K. Pokharel, P.R. Regmi, T.P. Dahal, S. Bhattarai, C.P. Pokheral, P. Gotame, T. Rayamajhi, R.C. Kandel \& A. Gurung (2021). Birds of Barandabhar Corridor Forest, Chitwan, Nepal. Journal of Threatened Taxa 13(11): 19509-19526. https://doi.org/10.11609/jott.6614.13.11.19509-19526

Copyright: @ Lamichhane et al. 2021. Creative Commons Attribution 4.0 International License. JoTT allows unrestricted use, reproduction, and distribution of this article in any medium by providing adequate credit to the author(s) and the source of publication.

Funding: UNDP-GEF Tiger and Rhino Landscape Conservation Project (2001-2012); Beeshazar and Associated Lakes Conservation and Community Empowerment Project (2015-2016).

Competing interests: The authors declare no competing interests.

Author details: Saneer Lamichhane, Santosh Bhattarai, Tulasi Prasad Dahal, Aashish Gurung: Conservation Officer, National Trust for Nature Conservation. Babu Ram Lamichhane: Office Incharge, National Trust for Nature Conservation-Biodiversity Conservation Center. Kapil Pokharel: Wildlife Technician, National Trust for Nature Conservation-Biodiversity Conservation Center. Pramod Raj Regmi: Natural Resource Conservation Assistant, National Trust for Nature Conservation. Chiranjibi Prasad Pokheral: Project Manager, National Trust for Nature Conservation. Pabitra Gotame: Ranger, Ministry of Forest and Environment, Government of Nepal. Trishna Rayamajhi: Graduate Student, Cornell University, USA. Ram Chandra Kandel: Secretary, Ministry of Industry, Tourism, Forests and Environment, Province No.2, Nepal

Author contributions: SL, BRL, RCK, CP: conceptualize the study; SL, BRL, TPD, SB, AG, KP, PG, TR: conducted the field survey of 2015 and 2016 and compiled the rest data; SL, BRL: finalized the manuscript; RCK, CP, BRL: supervised the study

Acknowledgements: We would like to acknowledge the financial support of UNDP - GEF (Tiger and Rhino Landscape Conservation project (project period: 2001-2012) and Beeshazar and Associated Lakes Conservation and Community Empowerment Project (project period: 2015-2016) to study the biodiversity of the Barandabhar Corridor Forest. We appreciate the support of Mr. Ram Kumar Aryal, former office-in-charge, NTNC-BCC to prepare the bird checklist. We also thank all the wildlife technicians of NTNC-BCC for their continuous risky frontline support in wildlife research.

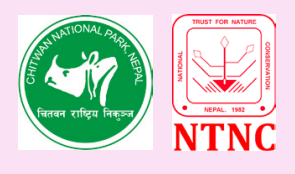




\section{INTRODUCTION}

Nepal is a biodiversity hotspot supporting $9.5 \%$ of the world's bird species, i.e., 886 species (DNPWC \& $B C N$ 2019). Birds are one of the most studied groups in Nepal, and information on birds is well documented in the form of field guides and reference books (Inskipp et al. 2013). The status and distribution of birds in Nepal has been studied by various researchers (e.g., Fleming et al. 1976; Inskipp \& Inskipp 1985; Cocker \& Inskipp 1988; Inskipp \& Inskipp 1991; Baral et al. 1996; Baral \& Inskipp 2004; Inskipp \& Inskipp 2012; Baral et al. 2012; Grimmett et al. 2016; Inskipp et al. 2017). In Nepal, wetland birds are the only vertebrate taxon that have been monitored every year since 1987. This survey has gained attention as the mid-winter water bird count (Baral 2009). Such long-term studies on birds and their associated habitats have become pivotal in the designation of Important Bird and Biodiversity Areas (IBAs) in Nepal. Nepal now has 32 IBAs listed (27 declared and 5 proposed with IBA codes) (BCN 2020). One of these IBAs is the Barandabhar Corridor Forest (BCF), which is important for bird conservation. Beeshazar and adjacent lakes are located to the south of the BCF and were designated as a Ramsar Site (Site no. 1313) in 2003.

The global loss of biodiversity is continuing at an alarming rate and increasing anthropogenic impacts are exacerbating the trend of species loss. Despite a significant increase in conservation activities, the rate of loss of species has not decreased (Butchart 2010; Schrauth \& Wink 2018). In addition, there are staggering losses of birds in the world (Dirzo 2014; Hallmann et al. 2017; Rosenberg 2019). To monitor and document environmental health and ecosystem integrity such as food availability, birds act as indicator species because they are easy to detect and observe, widely distributed, cover different levels of ecological pyramids, and the links among bird communities, vegetal associations and territory have been clearly demonstrated (Petty \& Avery 1990; Padoa-Schioppa et al. 2006; Schrauth \& Wink 2018; Rosenberg 2019). Hence, it is necessary for longterm monitoring and to keep baseline records of bird species. The previous studies by Adhikari et al. (2000, 2018) of birds and wetland birds in BCF were of short duration and have missed many bird species.

$B C F$, representing the IBA, lacks an updated checklist prepared from long-term monitoring of birds. Our study attempted to fill the gap of previous studies by providing the most recent data with revised nomenclature, vernacular names for public awareness including longterm data from 2002-2012 and 2015-2016 along with a checklist of birds of BCF from other studies after 2000. This checklist will serve as a reference for researchers, conservationists, planners, and bird enthusiasts.

\section{Study Area}

Barandabhar Corridor Forest (BCF) covers $87.9 \mathrm{~km}^{2}$ areas in the north of Chitwan National Park (CNP). It is the only remaining forest patch in Nepal that connects CNP with the Mahabharat range in the north (Bhattarai \& Basnet 2004; Lamichhane et al. 2018). BCF has a subtropical climate with winter, spring, and monsoon seasons (Bhattarai 2003). The area of BCF is dominated by Sal Shorea robusta forest and partly by riverine and mixed hardwood forest (Bhattarai 2003; NTNC 2003). This forest acts as a migratory route for different bird species (Adhikari et al. 2000, 2018).

The East-West highway (Mahendra Highway) bisects this corridor. The southern part falls under the buffer zone of Chitwan National Park and is guarded by the Nepali army (Lamichhane et al. 2018). Human disturbance is relatively low in the southern part. The northern part falls under the jurisdiction of the Divisional Forest Office, Chitwan and is managed as a protected forest. Despite its legal status of protected forest, human pressure in this part is relatively high due to the dependence of local communities on forest resources such as fodder, fuelwood, timber, grazing, NTFPs, and lack of strong enforcement. BCF is surrounded by heavily populated settlements of municipalities, namely, Ratnanagar Municipality on the east, Kalika on the north-east, and Bharatpur metropolitan city in the west (Figure 1). This has added human-pressure to this corridor and timely consideration for the conservation and management of $\mathrm{BCF}$ is needed.

\section{METHODS}

Bird species present in BCF were assessed by recording direct sightings. The survey was conducted by a team of experts and field staff of the National Trust for Nature Conservation (NTNC) in two-time frames: 2002-2012 and 2015-2016. NTNC in collaboration with the Divisional Forest Office and Chitwan National Park Office administered both surveys by mobilizing field technicians and researchers. Six survey routes of length $\sim 6$ to $11 \mathrm{~km}$ were set for the bird survey from 2002 to 2012. For 2015 to 2016, the number of transects were increased to 16 with a length of $\sim 4$ to $8 \mathrm{~km}$ and changed the orientation of the transects to a cross-section of the corridor (Figure 1). The transects were designed 


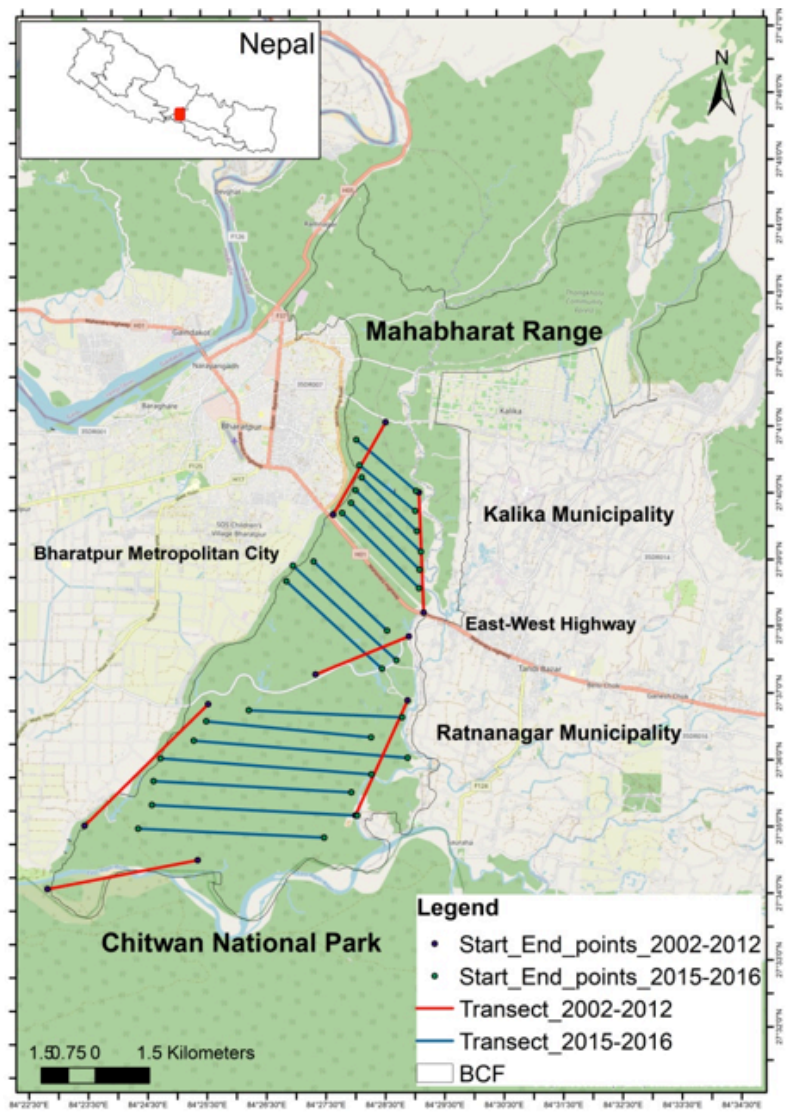

Figure 1. Transects set to identify bird species of Barandabhar Corridor Forest.

to cover all habitat types (wetlands, grasslands, open areas, forest) of BCF. At each starting point and at every $250 \mathrm{~m}$ throughout the transect, there was a minute disturbance pause and five-minute observation pause to sight the birds. Bushnell binoculars $(8 \times 42)$ were used to sight the birds. Every year, pre-monsoon (March-April) and post-monsoon (October-November) surveys were conducted to detect the summer and winter visitors, respectively. Since the activities of the birds are high in the morning period, we chose the survey time from 0700 to $0900 \mathrm{~h}$. Only one transect was surveyed each day to limit the research in the morning (0700-0900 h). For long transects ( $>4 \mathrm{~km}$ ), we either mobilized multiple groups of the surveyors (3-4 surveyors/group on foot) or the same team surveyed on multiple days depending on the length of the transect. For example, an 11-km transect was surveyed in three days (average $4 \mathrm{~km}$ transect survey in a day in two hours survey period). The survey in a transect was continued the next day from the point it was left in the previous day. The total number of bird species encountered was recorded in this period. We also collated the bird checklist previously published
(Adhikari et al. 2000, 2018) to prepare the updated bird checklist. We only included bird records after 2000.

\section{RESULTS}

BCF was found to support 372 bird species belonging to 20 orders and 80 families (Annexe 1) with a total survey effort of $1,506 \mathrm{~km}$. A total of 287 species was recorded during the survey of 2002-2012 and an additional 39 species were detected during 2015-2016. Twenty species recorded during 2002-2012 were not found during 2015-2016 surveys. In addition, the bird checklists of Adhikari et al. $(2000,2018)$ included 12 and 6 bird species, respectively, which were not recorded during our survey (2002-2012 and 2015-16) in Barandabhar. In November 2019, Great White Pelicans Pelecanus onocrotalus were spotted in BCF (Kathmandu Post 2019) which is also included in our checklist. Accipitridae family consisted of the highest number

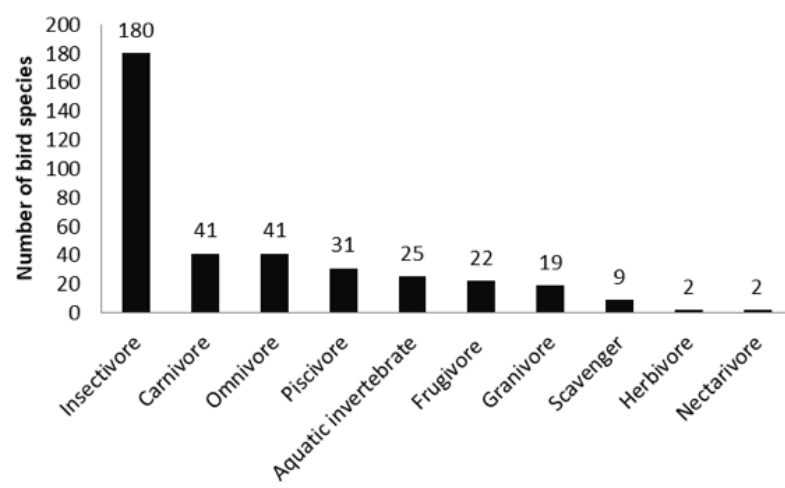

Figure 2. Foraging guild of bird species of Barandabhar Corridor Forest.

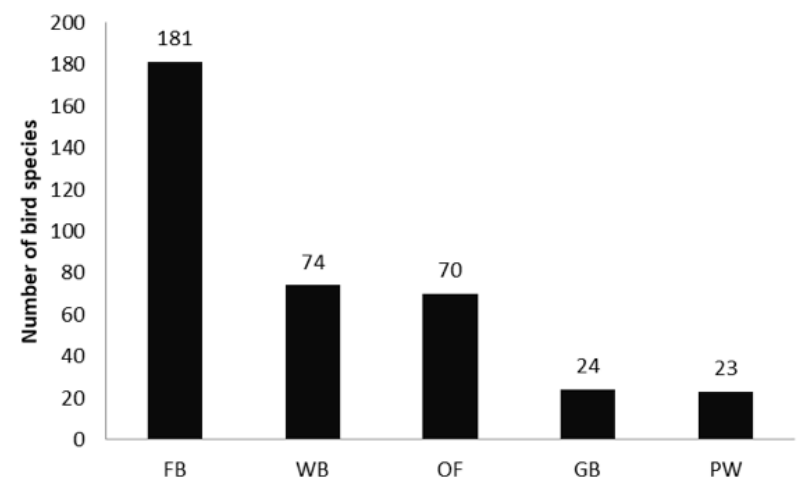

Figure 3. Habitat types and number of bird species of Barandabhar Corridor Forest. FB-Forest Bird | WB-Wetland bird | GBGrassland bird | OFB-Open Field bird | PW-Partially water bird (near water habitat). 
Table 1. IUCN status and migration group of bird species of Barandabhar Corridor Forest.

\begin{tabular}{|l|c|c|c|c|c|c|}
\hline $\begin{array}{l}\text { Migration } \\
\text { group }\end{array}$ & CR & EN & VU & NT & LC & Total \\
\hline Passage & & & & 2 & 9 & 11 \\
\hline Resident & 3 & & 6 & 10 & 215 & 234 \\
\hline $\begin{array}{l}\text { Summer } \\
\text { migratory }\end{array}$ & & & & & 28 & 28 \\
\hline $\begin{array}{l}\text { Winter } \\
\text { migratory }\end{array}$ & 2 & 3 & 2 & 3 & 89 & 99 \\
\hline Total & 5 & 3 & 8 & 15 & 341 & 372 \\
\hline
\end{tabular}

CR-Critically endangered | EN-Endangered | VU-Vulnerable | NT-Near Threatened | LC-Least Concern.

of species ( $n=32)$ followed by Muscicapidae $(n=30)$, and Anatidae ( $n=18)$. According to the foraging guild, insectivorous bird species were highest ( $n=180$ species) followed by equal number of carnivorous $(n=41)$ and omnivorous $(n=41)$ species (Figure 2 ). Out of the total bird species recorded, $62.9 \%, 26.6 \%, 7.5 \%$, and $2.9 \%$ of bird species of BCF are resident (R), winter migrants $(W)$, summer migrants (S), and passage migrants $(P)$, respectively (Table 1 ). The bird list also includes globally threatened species: five Critically Endangered, three Endangered, eight Vulnerable, and 15 Near Threatened species. In CITES enlisted categories, we recorded a single bird species each in CITES I and CITES III, and 53 bird species in CITES II category. According to the habitat type, there were 181 bird species of forest, 74 species of wetland, 24 species of grassland, 70 species of open field, and 23 species of partially wetland birds (Figure 3 ). The checklist is presented in Annexe 1.

\section{DISCUSSION}

Our study reported a high diversity of bird species in Barandabhar including 16 globally threatened species (Critically Endangered, Endangered, and Vulnerable) which further justifies the stronghold of its IBA status. The proximity to Chitwan National Park, one of the global biodiversity hotspots may have contributed to the high species diversity of birds. The southern portion (south of the highway of BCF) is the buffer zone of CNP and includes the Ramsar listed Beeshazar and associated lakes. CNP has a total of 544 recorded bird species (CNP 2019). As a contiguous habitat, the movement of the birds from CNP to adjoining BCF area is obvious.

Insectivorous bird species are dominant amongst the various species in BCF consisting of $48.38 \%$ of the total species. A recent study suggests that $40 \%$ of the insect species globally are in decline due to intensification of agriculture using a large volume of pesticides and fertilizers (Hallmann et al. 2017; Dougals 2019). Schrauth \& Wink (2018) stated that the decrease in insect abundance affects higher trophic levels like insectivorous birds. There is no cultivation inside BCF and human movements are also limited. We do not have data on the insect communities of BCF. However, we can hypothesize that the high number of insectivorous bird species in BCF means good insect communities are likely to be thriving in the area. Besides the insectivores, the carnivore and omnivore are the second largest group by species diversity (11\% each) in BCF.

A total of nine scavenger species was recorded from BCF, of which four are Critically Endangered, and among them three are resident. BCF is immediately surrounded by municipalities with agricultural fields and pasture for livestock. The scavengers are dependent on dead wildlife/ livestock in and around BCF. The use of diclofenac is detrimental to the survival of these scavengers (Oaks et al. 2004; Swan et al. 2006). So, a conservation awareness program to communities on the use of meloxicam which is an alternative for diclofenac would be a good solution for the survival of a viable population of scavengers (Swarup et al. 2007; Thapa 2009) in BCF.

BCF supports $62.9 \%$ resident, $26.6 \%$ winter migratory, $7.5 \%$ summer migratory, and $2.9 \%$ passage migrant birds. These migratory species have different breeding sites, wintering sites, and stopover sites or passage routes. These are sensitive species because a disturbance in any of these sites can cause a decline in their population. Since, BCF is a small biological corridor for migratory and passage birds, we must focus on to return the healthy breeding population so that they visit the area every year.

The transect used for the 2015-2016 surveys seems more effective because $10 \%$ more species were recorded in these two years compared to 2002-2012. Even so, the data lack the total species present in the survey of 20022012. This may be because the survey efforts of 20152016 covered $384 \mathrm{~km}$ compared to the survey efforts of $1,122 \mathrm{~km}$ during the 2002-2012 surveys.

One of the major challenges of the $21^{\text {st }}$ century is to globally reduce the rate of species loss (Barnosky et al. 2011; Pimm et al. 2014; Rosenberg 2019). In order to reduce threats to existing bird diversity in $B C F$, the government has endorsed the Barandabhar Corridor Forest Management Plan and the Beeshazari Lake Management Plan that aim to conserve the flora and fauna and their habitat in BCF. The proper implementation and timely revision of these existing 
plans and policies of BCF help to address the difficulties to protect bird diversity. Raising conservation awareness, removal of alien species, indigenous fish spawning in the wetlands, and incorporating birds as a separate chapter in management plans will help to sustain a healthy bird community in BCF.

This long-term data on bird species in BCF may act as the base-line for future reference, help in further research works, support managers for conservation efforts, and to record any changes in the composition of bird species of BCF. Despite BCF being divided into two portions, i.e., north and south portions, we believe the conservation intervention should be integrated and managed as a single ecological unit.

\section{REFERENCES}

Adhikari, J.N., B.P. Bhattarai \& T.B. Thapa (2018). Diversity and conservation threats of water birds in and around Barandabhar corridor forest, Chitwan, Nepal. Journal of Natural History Museum 30: 164-179.

Adhikari, R., R. Karmacharya, Y. Adhikari \& D.R. Sapkota (2000). The birds of Barandabhar. Bird Education Society. http://himalaya. socanth.cam.ac.uk/collections/inskipp/2000_003.pdf Electronic version accessed 21 July 2020.

Baral, H.S., U.R. Regmi, L.P. Poudyal \& R. Acharya (2012). Status and conservation of birds in Nepal. Biodiversity Conservation in Nepal: A Success Story. Department of National Parks and Wildlife Conservation, Kathmandu.

Baral, H.S. (2009). Updated status of Nepal's wetland birds. Banko Janakari February (special issue): 30-35. https://doi.org/10.3126/ banko.v19i3.2209

Baral, H.S. \& C. Inskipp (2005). Important Bird Areas in Nepal: key sites for conservation. Bird Conservation Nepal, 242pp.

Baral, H.S. \& C. Inskipp (2004). The state of Nepal's birds 2004. Kathmandu: Department of National Parks and Wildlife Conservation, Bird Conservation Nepal and IUCN Nepal.

Baral, H.S., C. Inskipp, T.P. Inskipp \& U.R. Regmi (1996). Threatened birds of Nepal. BCN and DNPWC, Bird Conservation Nepal and Department of National Parks and Wildlife Conservation, Kathmandu, 61-90pp.

Barnosky, A.D., N. Matzke, S. Tomiya, G.O.U. Wogan, B. Swartz, T.B. Quental, C. Marshall, J.L. McGuire, E.L. Lindsey, K.C. Maguire, B. Marsey \& E.A. Ferrer (2011). Has the Earth's sixth mass extinction already arrived? Nature 471(7336): 51-57. https://doi.org.1038/ nature09678

BCN (2020). Bird Conservation Nepal. https://www.birdlifenepal.org/ birds/important-birds-areas. Accessed 24 September 2020.

Bhattarai, B.P. (2003). Population Status and Conservation Threats of Wild Ungulates in Barandabhar Corridor Forest, Chitwan. M.Sc. thesis. Central Department of Zoology, Tribhuvan University, Kathmandu, Nepal.

Bhattarai, B.P. \& K. Basnet (2004). "Assessment of crop damage by wild ungulates in the eastern side of Barandabhar Corridor Forest, Chitwan.", pp. 23-26. In: Proceedings of IV National Conference on Science and Technology.

Butchart, S.H., M. Walpole, B. Collen, A. van Strien, J.P. Scharlemann, R.E. Almond, J.E.M. Baillie, B. Bomhard, C. Brown, J. Bruno \& K.E. Carpenter (2010). Global biodiversity decline continues. Science 328: 1164-1168. https://doi.org/10.1126/science.1187512

CNP (2019). https://www.chitwannationalpark.gov.np/. Electric version accessed 27 July 2020
Cocker, P.M. \& C. Inskipp (1988). A Himalayan ornithologist: the life and work of Brian Houghton Hodgson. Oxford University Press, Oxford, 98pp.

Dirzo, R., H.S. Young, M. Galetti, G. Ceballos, N.J. Isaac \& B. Collen (2014). Defaunation in the Anthropocene. Science 345: 401-406.

DNPWC \& BCN (2019). Birds of Nepal: An official checklist. Department of National Parks and Wildlife Conservation and Bird Conservation Kathmandu, Nepal.

Douglas, M. (2019). Why insect populations are plummetingand why it matters. https://www.nationalgeographic.com/ animals/2019/02/why-insect-populations-are-plummeting-andwhy-it-matters/. Electronic version accessed 21 July 2020.

Fleming, R.L. Sr, R.L. Jr. Fleming \& L.S. Bangdel (1976) Birds of Nepal. Flemings, Kathmandu, 349pp.

Grimmett, R., C. Inskipp, T. Inskipp \& H.S. Baral (2016). Birds of Nepal. $1^{\text {st }}$ Edition. Bloomsbury Publishing, 386pp.

Hallmann, C.A., M. Sorg, E. Jongejans, H. Siepel, N. Hofland, H. Schwan, W. Stenmans, A. Muller, H. Sumser, T. Hörren, D. Goulson \& H. da Kroon (2017). More than 75 percent decline over 27 years in total flying insect biomass in protected areas. PloS One 12: e0185809. https://doi.org/10.1371/journal.pone.0185809

India Biodiversity Portal (2020). https://indiabiodiversity.org. Accessed 19 July 2020.

Inskipp, C., H.S. Baral, T. Inskipp, A.P. Khatiwada, M.P. Khatiwada, L.P. Poudyal \& R. Amin (2017). Nepal's National Red List of Birds. Journal of Threatened Taxa 9(1): 9700-9722. https://doi.org/10.11609/ jott.2855.9.1.9700-9722

Inskipp, C., H.S. Baral, T. Inskipp \& A. Stattersfield (2013). The state of Nepal birds 2010. Journal of Threatened Taxa 5(1): 3473-3503. https://doi.org/10.11609/JoTT.03276.933

Inskipp, T. \& C. Inskipp ( 2012). Bibliography of birds of Nepal. Published on http://www.himalayannature.org/uploads/Nepal\%20 bibliography_17Feb.2012.pdf. Accessed 21 July 2020.

Inskipp, C. \& T. Inskipp (1991). A guide to the birds of Nepal. Second edition. Christopher Helm, London, UK. http://archive. org/details/guidetobirdsofne85insk. Accessed 21 July 2020. Inskipp, C. \& T. Inskipp (1985). A Guide to the Birds of Nepal. London, UK.

IUCN (2020). www.iucnredlist.org. Accessed on 20 June 2020.

Kathmmandu Post (2019). Migratory Great White Pelican spotted in Chitwan. https://kathmandupost.com/national/2017/04/21/ migratory-great-white-pelican-spotted-in-chitwan. Electronic version accessed 21 July 2020

Lamichhane, S., A. Gurung, C.P. Pokheral, T. Rayamajhi, P. Gotame, P.R. Regmi \& B.R. Lamichhane (2018). First record of the Dhole Cuon alpinus (Mammalia: Carnivora: Canidae) in Barandabhar Corridor Forest, Chitwan, Nepal. Journal of Threatened Taxa 10(1): 11243-11244. https://doi.org/10.11609/ jott.3533.10.1.11243-11244

NTNC (2003). Ecological assessment of Barandabhar Corridor Forest. Final Report. National Trust for Nature Conservation (NTNC), Biodiversity Conservation Center, Tiger, Rhino Conservation Project, Sauraha, Chitwan, Nepal.

Oaks, J.L., M. Gilbert, M.Z. Virani, R.T. Watson, C.U. Meteyer, B.A. Rideout, H.L. Shivaprasad, S. Ahmed, M.J.I Chaudhry, M. Arshad \& S. Mahmood (2004). Diclofenac residues as the cause of vulture population decline in Pakistan. Nature 427(6975): 630-633.

Padoa-Schioppa, E., M. Baietto, R. Massa \& L. Bottoni (2006). Bird communities as bioindicators: The focal species concept in agricultural landscapes. Ecological Indicators 6: 83-93.

Petty, S.J. \& M.I. Avery (1990). Forest Bird Communities. Occasional Papers 26. Forestry Commission, Edinburgh.

Pimm, S.L., C.N. Jenkins, R. Abell, T.M. Brooks, J.L. Gittleman, L.N. Joppa, P.H. Raven, C.M. Roberts \& J.O. Sexton (2014). The biodiversity of species and their rates of extinction, distribution, and protection. Science 344(6187): 124672. https://doi.org/10.1126/ science. 1246752

Rosenberg, K.V., A.M. Dokter, P.J. Blancher, J.R. Sauer, A.C. Smith, P.A. Smith, J.C. Stanton, A. Punjabi, L. Helft, M. Parr \& P.P. Marra (2019). 
Annexe 1. Checklist of the birds of BCF.

\begin{tabular}{|c|c|c|c|c|c|c|c|c|}
\hline & $\begin{array}{l}\text { Order/ Family/ Common } \\
\text { name }\end{array}$ & Scientific name & Nepali name & IUCN & CITES & $\begin{array}{l}\text { Foraging } \\
\text { guild }\end{array}$ & $\begin{array}{l}\text { Migration } \\
\text { group }\end{array}$ & $\begin{array}{c}\text { Habitat } \\
\text { Type }\end{array}$ \\
\hline & ACCIPITRIFORMES & & & & & & & \\
\hline & Accipitridae & & & & & & & \\
\hline 1 & Besra & $\begin{array}{l}\text { Accipiter virgatus (Temminck, } \\
\text { 1822) }\end{array}$ & बेसरा & LC & II & Carnivore & $\mathrm{R}$ & $\mathrm{FB}$ \\
\hline 2 & Black Baza & $\begin{array}{l}\text { Aviceda leuphotes (Dumont, } \\
\text { 1820) }\end{array}$ & गोमायु महाचील & LC & II & Carnivore & $\mathrm{S}$ & $\mathrm{FB}$ \\
\hline 3 & Black Eagle & $\begin{array}{l}\text { Ictinaetus malayensis (Temminck, } \\
\text { 1822) }\end{array}$ & द्रोणक चील & LC & II & Carnivore & $\mathrm{R}$ & $\mathrm{FB}$ \\
\hline 4 & Black Kite & Milvus migrans (Boddaert, 1783) & कालो चील & LC & ॥ & Scavenger & W & OF \\
\hline 5 & Black-winged Kite & $\begin{array}{l}\text { Elanus caeruleus (Desfontaines, } \\
\text { 1789) }\end{array}$ & मुसे चील & LC & ॥ & Carnivore & $\mathrm{R}$ & OF \\
\hline 6 & Booted Eagle & $\begin{array}{l}\text { Hieraaetus pennatus (Gmelin, } \\
1788 \text { ) }\end{array}$ & काँडचन्द्र चील & LC & II & Carnivore & W & $\mathrm{FB}$ \\
\hline 7 & Changeable Hawk-eagle & $\begin{array}{l}\text { Nisaetus cirrhatus\# (Gmelin, } \\
\text { 1788) }\end{array}$ & सदल चील & LC & II & Carnivore & $\mathrm{R}$ & $\mathrm{FB}$ \\
\hline 8 & Cinereous Vulture & $\begin{array}{l}\text { Aegypius monachus (Linnaeus, } \\
1766 \text { ) }\end{array}$ & राज गिद्द & NT & II & Scavenger & w & $\mathrm{FB}$ \\
\hline 9 & Crested Goshawk & $\begin{array}{l}\text { Accipiter trivirgatus (Temminck, } \\
\text { 1824) }\end{array}$ & कल्की बाज & LC & ॥ & Carnivore & $\mathrm{R}$ & $\mathrm{FB}$ \\
\hline 10 & Crested Serpent-eagle & Spilornis cheela(Latham 1790) & काकाकुल & LC & ॥ & Carnivore & $\mathrm{R}$ & $\mathrm{FB}$ \\
\hline 11 & Egyptian Vulture & $\begin{array}{l}\text { Neophron percnopterus } \\
\text { (Linnaeus, 1758) }\end{array}$ & सेतो गिद्द & EN & II & Scavenger & W & OF \\
\hline 12 & Eurasian Buzzard & Buteo buteo (Linnaeus, 1758) & श्येनबाज & LC & ॥ & Carnivore & w & $\mathrm{FB}$ \\
\hline 13 & Eurasian Sparrowhawk & Accipiter nisus (Linnaeus, 1758) & बनबाज & LC & ॥ & Carnivore & w & $\mathrm{FB}$ \\
\hline 14 & Greater Spotted Eagle & Clanga clanga (Pallas, 1811) & जोबहर महाचील & VU & ॥ & Carnivore & w & OF \\
\hline 15 & Grey-headed Fish-eagle & $\begin{array}{l}\text { Icthyophaga ichthyaetus\# } \\
\text { (Horsfield, 1821) }\end{array}$ & माछाकुल & NT & ॥ & Piscivore & $\mathrm{R}$ & PW \\
\hline 16 & Griffon Vulture & Gyps fulvus(Hablitz, 1783) & खैरो गिद्द & LC & II & Scavenger & w & OF \\
\hline 17 & Hen Harrier & Circus cyaneus (Linnaeus, 1766) & चल्लाचोर भुइँचील & LC & II & Carnivore & w & OF \\
\hline 18 & Himalayan Griffon & Gyps himalayensis (Hume, 1869) & हिमाली गिद्द & NT & ॥ & Scavenger & $\mathrm{R}$ & OF \\
\hline 19 & Indian Spotted Eagle & Clanga hastata (Lesson, 1834) & लघु महाचील & VU & II & Carnivore & $\mathrm{R}$ & OF \\
\hline 20 & Indian Vulture & Gyps indicus* (Scopoli, 1786) & लामो ठूँडे गिद्द & $C R$ & ॥ & Scavenger & $\mathrm{R}$ & OF \\
\hline 21 & Long-legged Buzzard & Buteo rufinus (Cretzschmar, 1829) & लाम्बुट्टे स्यनबाज & LC & II & Carnivore & w & $\mathrm{FB}$ \\
\hline 22 & Oriental Honey-buzzard & $\begin{array}{l}\text { Pernis ptilorhyncus (Temminck, } \\
\text { 1821) }\end{array}$ & मधु चील & LC & ॥ & Carnivore & $\mathrm{R}$ & $\mathrm{FB}$ \\
\hline 23 & Pallas's Fish-eagle & $\begin{array}{l}\text { Haliaeetus leucoryphus (Pallas, } \\
\text { 1771) }\end{array}$ & बोक्सी चील & EN & ॥ & Piscivore & w & PW \\
\hline 24 & Pied Harrier & $\begin{array}{l}\text { Circus melanoleucos (Pennant, } \\
1769 \text { ) }\end{array}$ & माले भुइंचील & LC & ॥ & Carnivore & $\mathrm{R}$ & OF \\
\hline 25 & Red-headed Vulture & Sarcogyps calvus (Scopoli, 1786) & सुन गिद्द & $C R$ & II & Scavenger & w & OF \\
\hline 26 & Shikra & Accipiter badius (Gmelin, 1788) & सिक्रा & LC & ॥ & Carnivore & $\mathrm{R}$ & $\mathrm{FB}$ \\
\hline 27 & Short-toed Snake Eagle & Circaetus gallicus* (Gmelin, 1788) & सर्पहरि चील & LC & II & Carnivore & $P$ & $\mathrm{FB}$ \\
\hline 28 & Slender-billed Vulture & Gyps tenuirostris (Gray, 1844) & सानो खैरो गिद्द & $C R$ & ॥ & Scavenger & $\mathrm{R}$ & OF \\
\hline 29 & Steppe Eagle & Aquila nipalensis (Hodgson, 1833) & गोमायु महाचील & EN & ॥ & Carnivore & w & $\mathrm{FB}$ \\
\hline 30 & White-eyed Buzzard & Butastur teesa (Franklin, 1831) & जमल श्वेतबाज & LC & ॥ & Carnivore & $\mathrm{R}$ & $\mathrm{FB}$ \\
\hline 31 & White-rumped Vulture & Gyps bengalensis (Gmelin, 1788) & डड़र गिद्द & $C R$ & ॥ & Scavenger & $\mathrm{R}$ & OF \\
\hline \multirow[t]{2}{*}{32} & White-tailed Sea-eagle & $\begin{array}{l}\text { Haliaeetus albicilla (Linnaeus, } \\
\text { 1758) }\end{array}$ & कंगम चिल & LC & ॥ & Carnivore & $\mathrm{R}$ & PW \\
\hline & Pandionidae & & & & & & & \\
\hline \multirow[t]{3}{*}{33} & Osprey & $\begin{array}{l}\text { Pandion haliaetus (Linnaeus, } \\
1758 \text { ) }\end{array}$ & मलाहा चील & LC & ॥ & Piscivore & $\mathrm{R}$ & PW \\
\hline & ANSERIFORMES & & & & & & & \\
\hline & Anatidae & & & & & & & \\
\hline 34 & Common Goldeneye & $\begin{array}{l}\text { Bucephala clangula (Linnaeus, } \\
\text { 1758) }\end{array}$ & स्वर्ण नयन् हाँस & LC & - & Omnivore & $P$ & WB \\
\hline
\end{tabular}




\begin{tabular}{|c|c|c|c|c|c|c|c|c|}
\hline & $\begin{array}{l}\text { Order/ Family/ Common } \\
\text { name }\end{array}$ & Scientific name & Nepali name & IUCN & CITES & $\begin{array}{l}\text { Foraging } \\
\text { guild }\end{array}$ & $\begin{array}{l}\text { Migration } \\
\text { group }\end{array}$ & $\begin{array}{c}\text { Habitat } \\
\text { Type }\end{array}$ \\
\hline 35 & Common Pochard & Aythya ferina (Linnaeus, 1758) & कैलो टाउके हाँस & vu & - & Carnivore & w & WB \\
\hline 36 & Common Teal & Anas crecca (Linnaeus, 1758) & बिजुलागईरी & LC & - & Omnivore & W & WB \\
\hline 37 & Cotton Pygmy-goose & $\begin{array}{l}\text { Nettapus coromandelianus } \\
\text { (Gmelin, 1789) }\end{array}$ & हरि हाँस & LC & - & Omnivore & W & WB \\
\hline 38 & Eurasian Wigeon & $\begin{array}{l}\text { Mareca penelope (Linnaeus, } \\
\text { 1758) }\end{array}$ & सिन्दुरे हाँस & LC & - & Omnivore & W & WB \\
\hline 39 & Ferruginous Duck & $\begin{array}{l}\text { Aythya nyroca (Guldenstadt, } \\
\text { 1770) }\end{array}$ & मालक हाँस & NT & - & Omnivore & W & WB \\
\hline 40 & Gadwall & $\begin{array}{l}\text { Mareca strepera\# (Linnaeus, } \\
1758 \text { ) }\end{array}$ & खड्खडे हाँस & LC & - & Omnivore & W & WB \\
\hline 41 & Garganey & $\begin{array}{l}\text { Spatula querquedula \$ (Linnaeus, } \\
\text { 1758) }\end{array}$ & श्वेताआँखीभौं & LC & - & Omnivore & $\mathrm{R}$ & WB \\
\hline 42 & Goosander & $\begin{array}{l}\text { Mergus merganser* (Linnaeus, } \\
\text { 1758) }\end{array}$ & मणितुण्डक & LC & - & Piscivore & W & WB \\
\hline 43 & Indian Spot-billed Duck & $\begin{array}{l}\text { Anas poecilorhyncha* (Forster, } \\
\text { 1781) }\end{array}$ & नदुन हाँस & LC & - & Omnivore & W & WB \\
\hline 44 & Lesser Whistling Duck & $\begin{array}{l}\text { Dendrocygna javanica (Horsfield, } \\
\text { 1821) }\end{array}$ & सिलसिले & LC & - & Herbivore & $\mathrm{R}$ & WB \\
\hline 45 & Mallard & $\begin{array}{l}\text { Anas platyrhynchos (Linnaeus, } \\
\text { 1758) }\end{array}$ & हरियो टाउके हाँस & LC & - & Omnivore & W & WB \\
\hline 46 & Northern Pintail & Anas acuta (Linnaeus, 1758) & सुईरोपुच्छे & LC & - & Omnivore & w & WB \\
\hline 47 & Northern Shoveler & Spatula clypeata (Linnaeus, 1758) & बेल्चा ठुडे हाँस & LC & - & Omnivore & $P$ & WB \\
\hline 48 & Red-crested Pochard & Netta rufina (Pallas, 1773) & सुनजुरे हाँस & LC & - & Omnivore & w & WB \\
\hline 49 & Ruddy Shelduck & Tadorna ferruginea (Pallas, 1764) & चखेवा & LC & - & Omnivore & W & WB \\
\hline 50 & Smew & $\begin{array}{l}\text { Mergellus albellus (Linnaeus, } \\
\text { 1758) }\end{array}$ & देवदुत माणीतुन्दक & LC & - & Omnivore & W & WB \\
\hline \multirow[t]{3}{*}{51} & Tufted Duck & Aythya fuligula (Linnaeus, 1758) & कालीजुरे हाँस & LC & - & Carnivore & w & WB \\
\hline & APODIFORMES & & & & & & & \\
\hline & Hemiprocnidae & & & & & & & \\
\hline \multirow[t]{3}{*}{52} & Crested Treeswift & $\begin{array}{l}\text { Hemiprocne coronata (Tickell, } \\
\text { 1833) }\end{array}$ & जुरे गौंथली & LC & - & Insectivore & $\mathrm{R}$ & $\mathrm{FB}$ \\
\hline & BUCEROTIFORMES & & & & & & & \\
\hline & Bucerotidae & & & & & & & \\
\hline 53 & Great Hornbill & Buceros bicornis (Linnaeus, 1758) & राज धनेश & Vu & 1 & Frugivore & $\mathrm{R}$ & $\mathrm{FB}$ \\
\hline 54 & Indian Grey Hornbill & Ocyceros birostris (Scopoli, 1786) & खैरो धनेश & LC & - & Omnivore & $\mathrm{R}$ & $\mathrm{FB}$ \\
\hline \multirow[t]{2}{*}{55} & Oriental Pied Hornbill & $\begin{array}{l}\text { Anthracoceros albirostris (Shaw \& } \\
\text { Nodder, 1807) }\end{array}$ & कालो धनेश & LC & II & Frugivore & $\mathrm{R}$ & $\mathrm{FB}$ \\
\hline & Upupidae & & & & & & & \\
\hline \multirow[t]{3}{*}{56} & Common Hoopoe & Upupa epops (Linnaeus, 1758) & फाप्रे चरा ( दशैं चरा ) & LC & - & Insectivore & $\mathrm{R}$ & OF \\
\hline & CAPRIMULGIFORMES & & & & & & & \\
\hline & Apodidae & & & & & & & \\
\hline 57 & Alpine Swift & $\begin{array}{l}\text { Tachymarptis melba* (Linnaeus, } \\
\text { 1758) }\end{array}$ & बतासी गौँथली & LC & - & Insectivore & W & OF \\
\hline 58 & Himalayan Swiftlet & $\begin{array}{l}\text { Aerodramus brevirostris\# } \\
\text { (Horsfield, 1840) }\end{array}$ & धिचिका गौंथली & LC & - & Insectivore & W & OF \\
\hline 59 & House Swift & $\begin{array}{l}\text { Apus nipalensis \# (Hodgson, } \\
\text { 1837) }\end{array}$ & फिरिंरे घर गौंथली & LC & - & Insectivore & $\mathrm{R}$ & OF \\
\hline 60 & Little Swift & Apusaffinis@ (JE Gray, 1830) & फिफिरे घर गौंथली & LC & - & Insectivore & $\mathrm{R}$ & OF \\
\hline 61 & Pacific Swift & Apuspacificus@ (Latham, 1801) & पुछुरकापे गौथली & LC & - & Insectivore & $\mathrm{R}$ & OF \\
\hline 62 & Silver-backed Needletail & $\begin{array}{l}\text { Hirundapus cochinchinensis } \\
\text { (Oustalet, 1878) }\end{array}$ & चण्डी ढाडे गौंथली & LC & - & Insectivore & R & OF \\
\hline \multirow[t]{2}{*}{63} & White-rumped Spinetail & $\begin{array}{l}\text { Zoonavena sylvatica (Tickell, } \\
\text { 1846) }\end{array}$ & सानो वन गौंथली & LC & - & Insectivore & $\mathrm{R}$ & OF \\
\hline & Caprimulgidae & & & & & & & \\
\hline 64 & Large-tailed Nightjar & $\begin{array}{l}\text { Caprimulgus macrurus (Horsfield, } \\
\text { 1821) }\end{array}$ & लाम्पुछे चैते चरा & LC & - & Insectivore & $\mathrm{R}$ & $\mathrm{FB}$ \\
\hline 65 & Savanna Nightjar & $\begin{array}{l}\text { Caprimulgus affinis (Horsfield, } \\
\text { 1821) }\end{array}$ & चुइयाँ चैते चरा & LC & - & Insectivore & $\mathrm{R}$ & GB \\
\hline
\end{tabular}




\begin{tabular}{|c|c|c|c|c|c|c|c|c|}
\hline & $\begin{array}{l}\text { Order/ Family/ Common } \\
\text { name }\end{array}$ & Scientific name & Nepali name & IUCN & CITES & $\begin{array}{l}\text { Foraging } \\
\text { guild }\end{array}$ & $\begin{array}{l}\text { Migration } \\
\text { group }\end{array}$ & $\begin{array}{l}\text { Habitat } \\
\text { Type }\end{array}$ \\
\hline & CHARADRIIFORMES & & & & & & & \\
\hline & Burhinidae & & & & & & & \\
\hline \multirow[t]{2}{*}{66} & Eurasian Thick-knee & $\begin{array}{l}\text { Burhinus oedicnemus (Linnaeus, } \\
\text { 1758) }\end{array}$ & बगरबट्टाई & LC & - & Insectivore & $\mathrm{R}$ & WB \\
\hline & Charadriidae & & & & & & & \\
\hline 67 & Grey-headed Lapwing & Vanellus cinereus (Blyth, 1842) & खरानी टाउके हुटिट्याउँ & LC & - & $\begin{array}{c}\text { Aquatic } \\
\text { invertebrates }\end{array}$ & W & WB \\
\hline 68 & Kentish Plover & $\begin{array}{l}\text { Charadrius alexandrinus } \\
\text { (Linnaeus, 1758) }\end{array}$ & अलकचन्द्र राजपुत्रीका & LC & - & Carnivore & W & WB \\
\hline 69 & Little Ringed Plover & Charadrius dubius (Scopoli, 1786) & लघु राजपुत्रीका & LC & - & $\begin{array}{c}\text { Aquatic } \\
\text { invertebrates }\end{array}$ & $\mathrm{R}$ & WB \\
\hline 70 & Northern Lapwing & $\begin{array}{l}\text { Vanellus vanellus (Linnaeus, } \\
\text { 1758) }\end{array}$ & जुरे हुटिट्याउ & NT & - & $\begin{array}{c}\text { Aquatic } \\
\text { invertebrates }\end{array}$ & W & WB \\
\hline 71 & Pacific Golden Plover & Pluvialis fulva (Gmelin, 1789) & प्रसान्त सर्षपी & LC & - & $\begin{array}{c}\text { Aquatic } \\
\text { invertebrates }\end{array}$ & $P$ & WB \\
\hline 72 & Red-wattled Lapwing & Vanellus indicus (Boddaert, 1783) & हुटिट्याउँ & LC & - & $\begin{array}{c}\text { Aquatic } \\
\text { invertebrates }\end{array}$ & W & WB \\
\hline 73 & River Lapwing & Vanellus duvaucelii (Lesson, 1826) & खोले हुटिट्याजँ & NT & - & $\begin{array}{c}\text { Aquatic } \\
\text { invertebrates }\end{array}$ & $\mathrm{R}$ & WB \\
\hline \multirow[t]{2}{*}{74} & Yellow-wattled Lapwing & $\begin{array}{l}\text { Vanellus malabaricus (Boddaert, } \\
\text { 1783) }\end{array}$ & पहेंलो गाले हुटिट्याउँ & LC & - & Insectivore & $\mathrm{R}$ & WB \\
\hline & Glareolidae & & & & & & & \\
\hline \multirow[t]{2}{*}{75} & Small Pratincole & Glareola lactea (Temminck, 1820) & पानी गौंथली & LC & - & Insectivore & $\mathrm{R}$ & PW \\
\hline & Haematopodidae & & & & & & & \\
\hline \multirow[t]{2}{*}{76} & Eurasian Oystercatcher & $\begin{array}{l}\text { Haematopus ostralegus } \\
\text { (Linnaeus, 1758) }\end{array}$ & सिपि चरा & NT & - & $\begin{array}{c}\text { Aquatic } \\
\text { invertebrates }\end{array}$ & $P$ & WB \\
\hline & Jacanidae & & & & & & & \\
\hline 77 & Bronze-winged Jacana & $\begin{array}{l}\text { Metopidius indicus (Latham, } \\
\text { 1790) }\end{array}$ & कमल चरी & LC & - & $\begin{array}{c}\text { Aquatic } \\
\text { invertebrates }\end{array}$ & $\mathrm{R}$ & WB \\
\hline \multirow[t]{2}{*}{78} & Pheasant-tailed Jacana & $\begin{array}{l}\text { Hydrophasianus chirurgus } \\
\text { (Scopoli, 1786) }\end{array}$ & जलअपसरा & LC & - & Omnivore & $\mathrm{R}$ & WB \\
\hline & Laridae & & & & & & & \\
\hline 79 & Common Tern & Sterna hirundo (Linnaeus, 1758) & वायु फ्यालफ्याले & LC & - & Piscivore & $\mathrm{P}$ & PW \\
\hline \multirow[t]{2}{*}{80} & River Tern & Sterna aurantia (J.E. Gray, 1831) & कुरारी फ्यालफ्याले & NT & - & Carnivore & $\mathrm{R}$ & PW \\
\hline & Recurvirostridae & & & & & & & \\
\hline \multirow[t]{2}{*}{81} & Black-winged Stilt & $\begin{array}{l}\text { Himantopus himantopus } \\
\text { (Linnaeus, 1758) }\end{array}$ & प्रवालपाद & LC & - & $\begin{array}{c}\text { Aquatic } \\
\text { invertebrates }\end{array}$ & $\mathrm{P}$ & WB \\
\hline & Rostratulidae & & & & & & & \\
\hline \multirow[t]{2}{*}{82} & Greater Painted-snipe & $\begin{array}{l}\text { Rostratula benghalensis } \\
\text { (Linnaeus, 1758) }\end{array}$ & चित्राड़द & LC & - & Omnivore & $\mathrm{R}$ & WB \\
\hline & Scolopacidae & & & & & & & \\
\hline 83 & Black-tailed Godwit & Limosa limosa (Linnaeus, 1758) & मलगुजा & NT & - & Carnivore & $\mathrm{P}$ & WB \\
\hline 84 & Common Greenshank & $\begin{array}{l}\text { Tringa nebularia (Gunnerus, } \\
\text { 1767) }\end{array}$ & टिमटिमा & LC & - & $\begin{array}{c}\text { Aquatic } \\
\text { invertebrates }\end{array}$ & W & WB \\
\hline 85 & Common Redshank & Tringa totanus (Linnaeus, 1758) & लालखुट्टे टिमटिमा & LC & - & $\begin{array}{c}\text { Aquatic } \\
\text { invertebrates }\end{array}$ & W & WB \\
\hline 86 & Common Sandpiper & $\begin{array}{l}\text { Actitis hypoleucos (Linnaeus, } \\
1758 \text { ) }\end{array}$ & चन्चले सुढसुढिया & LC & - & $\begin{array}{c}\text { Aquatic } \\
\text { invertebrates }\end{array}$ & W & WB \\
\hline 87 & Common Snipe & $\begin{array}{l}\text { Gallinago gallinago (Linnaeus, } \\
\text { 1758) }\end{array}$ & पानी चाहा & LC & - & $\begin{array}{c}\text { Aquatic } \\
\text { invertebrates }\end{array}$ & W & WB \\
\hline 88 & Eurasian Woodcock & $\begin{array}{l}\text { Scolopax rusticola (Linnaeus, } \\
1758 \text { ) }\end{array}$ & ठूलो चाहा & LC & - & Insectivore & W & WB \\
\hline 89 & Green Sandpiper & Tringa ochropus (Linnaeus, 1758) & रूख सुढसुढिया & LC & - & $\begin{array}{c}\text { Aquatic } \\
\text { invertebrates }\end{array}$ & W & WB \\
\hline 90 & Little Stint & Calidris minuta (Leisler, 1812) & काली खुट्टे जलरड़ & LC & - & $\begin{array}{c}\text { Aquatic } \\
\text { invertebrates }\end{array}$ & W & WB \\
\hline 91 & Pintail Snipe & $\begin{array}{l}\text { Gallinago stenura (Bonaparte, } \\
\text { 1831) }\end{array}$ & भरक्चाहा & LC & - & $\begin{array}{c}\text { Aquatic } \\
\text { invertebrates }\end{array}$ & W & WB \\
\hline 92 & Temminck's Stint & Calidris temminckii (Leisler, 1812) & जलरझ़ & LC & - & $\begin{array}{c}\text { Aquatic } \\
\text { invertebrates }\end{array}$ & W & WB \\
\hline
\end{tabular}




\begin{tabular}{|c|c|c|c|c|c|c|c|c|}
\hline & $\begin{array}{l}\text { Order/ Family/ Common } \\
\text { name }\end{array}$ & Scientific name & Nepali name & IUCN & CITES & $\begin{array}{l}\text { Foraging } \\
\text { guild }\end{array}$ & $\begin{array}{l}\text { Migration } \\
\text { group }\end{array}$ & $\begin{array}{c}\text { Habitat } \\
\text { Type }\end{array}$ \\
\hline \multirow[t]{2}{*}{93} & Wood Sandpiper & Tringa glareola (Linnaeus, 1758) & वन सुढसुढिया & LC & - & $\begin{array}{c}\text { Aquatic } \\
\text { invertebrates }\end{array}$ & $P$ & WB \\
\hline & Turnicidae & & & & & & & \\
\hline \multirow[t]{3}{*}{94} & Common Buttonquail & $\begin{array}{l}\text { Turnix sylvatica (Desfontaines, } \\
\text { 1789) }\end{array}$ & सानो गट्टेबट्टाई & LC & - & Granivore & $\mathrm{R}$ & OF \\
\hline & CICONIIFORMES & & & & & & & \\
\hline & Ciconiidae & & & & & & & \\
\hline 95 & Asian Openbill & $\begin{array}{l}\text { Anastomus oscitans (Boddaert, } \\
\text { 1783) }\end{array}$ & चिम्टे गरुड & LC & - & Piscivore & W & WB \\
\hline 96 & Black Stork & Ciconia nigra (Linnaeus, 1758) & कालो गरुड & LC & II & Piscivore & W & WB \\
\hline 97 & Black-necked Stork & $\begin{array}{l}\text { Ephippiorhynchus asiaticus* } \\
\text { (Latham, 1790) }\end{array}$ & कृष्णकण्ठ गरुड & NT & - & Piscivore & $\mathrm{R}$ & WB \\
\hline 98 & Lesser Adjutant & $\begin{array}{l}\text { Leptoptilos javanicus (Horsfield, } \\
\text { 1821) }\end{array}$ & भुंडिफोर गरुड & VU & - & Piscivore & $\mathrm{R}$ & WB \\
\hline \multirow[t]{3}{*}{99} & $\begin{array}{l}\text { Asian Woolly-necked } \\
\text { Stork }\end{array}$ & $\begin{array}{l}\text { Ciconia episcopus (Boddaert, } \\
1783 \text { ) }\end{array}$ & $\begin{array}{l}\text { कपाशकण्ठ गरुड / लो } \\
\text { भिपापी }\end{array}$ & VU & - & Piscivore & $\mathrm{R}$ & WB \\
\hline & COLUMBIFORMES & & & & & & & \\
\hline & Columbidae & & & & & & & \\
\hline 100 & Common Wood Pigeon & $\begin{array}{l}\text { Columba palumbus (Linnaeus, } \\
\text { 1758) }\end{array}$ & डूलो वनपरेवा & LC & - & Granivore & W & $\mathrm{FB}$ \\
\hline 101 & Spotted-dove & $\begin{array}{l}\text { Spilopelia chinensis\# (Scopoli, } \\
\text { 1768) }\end{array}$ & कुर्ले ढुकुर & LC & - & Granivore & $\mathrm{R}$ & OF \\
\hline 102 & Eurasian Collared-dove & $\begin{array}{l}\text { Streptopelia decaocto } \\
\text { (Frivaldszky, 1838) }\end{array}$ & कण्ठे ढुकुर & LC & - & Granivore & $\mathrm{R}$ & OF \\
\hline 103 & $\begin{array}{l}\text { Grey-capped Emerald } \\
\text { Dove }\end{array}$ & $\begin{array}{l}\text { Chalcophaps indica (Linnaeus, } \\
\text { 1758) }\end{array}$ & सुन ढुकुर & LC & - & Frugivore & $\mathrm{R}$ & $\mathrm{FB}$ \\
\hline 104 & $\begin{array}{l}\text { Orange-breasted Green- } \\
\text { pigeon }\end{array}$ & Treron bicinctus (Jerdon, 1840) & सुन्तलेछाती हलेसो & LC & - & Frugivore & $\mathrm{R}$ & FB \\
\hline 105 & Oriental Turtle-dove & $\begin{array}{l}\text { Streptopelia orientalis (Latham, } \\
\text { 1790) }\end{array}$ & तुम्मे ढुकुर & LC & - & Granivore & W & OF \\
\hline 106 & Red Turtle-dove & $\begin{array}{l}\text { Streptopelia tranquebarica } \\
\text { (Hermann, 1804) }\end{array}$ & तामे ढुकुर & LC & - & Granivore & $\mathrm{R}$ & OF \\
\hline 107 & Sri Lanka Green-pigeon & $\begin{array}{l}\text { Treron pompadora (Gmelin, } \\
\text { 1789) }\end{array}$ & फुस्रो टाउके हलेसो & LC & - & Frugivore & $\mathrm{R}$ & $\mathrm{FB}$ \\
\hline 108 & $\begin{array}{l}\text { Thick-Billed Green- } \\
\text { pigeon }\end{array}$ & $\begin{array}{l}\text { Treron curvirostra@ (Gmelin, } \\
\text { 1789) }\end{array}$ & मोटोठुँडे हलेसो & LC & - & Insectivore & $\mathrm{R}$ & $\mathrm{FB}$ \\
\hline \multirow[t]{3}{*}{109} & $\begin{array}{l}\text { Yellow-footed Green- } \\
\text { pigeon }\end{array}$ & $\begin{array}{l}\text { Treron phoenicoptera (Latham, } \\
\text { 1790) }\end{array}$ & ठूलो हलेसो & LC & - & Frugivore & $\mathrm{R}$ & FB \\
\hline & CORACIIFORMES & & & & & & & \\
\hline & Alcedinidae & & & & & & & \\
\hline 110 & Black-capped Kingfisher & Halcyon pileata (Boddaert, 1783) & कालोटाऊके माटीकोरे & LC & - & Piscivore & W & PW \\
\hline 111 & Blue-eared Kingfisher & $\begin{array}{l}\text { Alcedo meninting (Horsfield, } \\
\text { 1821) }\end{array}$ & निलकर्ण माटीकोरे & LC & - & Piscivore & $\mathrm{R}$ & PW \\
\hline 112 & Common Kingfisher & Alcedo atthis (Linnaeus, 1758) & सानो माटीकोरे & LC & - & Piscivore & $\mathrm{R}$ & PW \\
\hline 113 & Pied Kingfisher & Ceryle rudis (Linnaeus, 1758) & छिरबिरे माटिकोरे & LC & - & Piscivore & $\mathrm{R}$ & PW \\
\hline 114 & Stork-billed Kingfisher & $\begin{array}{l}\text { Pelargopsis capensis (Linnaeus, } \\
1766 \text { ) }\end{array}$ & ठूलो माटीकोरे & LC & - & Piscivore & $\mathrm{R}$ & PW \\
\hline \multirow[t]{2}{*}{115} & $\begin{array}{l}\text { White-breasted } \\
\text { Kingfisher }\end{array}$ & $\begin{array}{l}\text { Halcyon smyrnensis (Linnaeus, } \\
\text { 1758) }\end{array}$ & सेतोकण्ठे माटीकोरे & LC & - & Piscivore & $\mathrm{R}$ & PW \\
\hline & Coraciidae & & & & & & & \\
\hline 116 & Indian Roller & $\begin{array}{l}\text { Coracias benghalensis (Linnaeus, } \\
1758 \text { ) }\end{array}$ & ठेऊवा & LC & - & Insectivore & $\mathrm{R}$ & OF \\
\hline \multirow[t]{2}{*}{117} & Oriental Dollarbird & $\begin{array}{l}\text { Eurystomus orientalis (Linnaeus, } \\
1766 \text { ) }\end{array}$ & निलठेऊवा & LC & - & Insectivore & S & $\mathrm{FB}$ \\
\hline & Meropidae & & & & & & & \\
\hline 118 & Green Bee-eater & Merops orientalis (Latham, 1801) & मुरली चरा & LC & - & Insectivore & R & OF \\
\hline 119 & Blue-bearded Bee-eater & $\begin{array}{l}\text { Nyctyornis athertoni (Jardine \& } \\
\text { Selby, 1830) }\end{array}$ & निलकण्ठ मुरली चरा & LC & - & Insectivore & $\mathrm{R}$ & FB \\
\hline 120 & Blue-tailed Bee-eater & $\begin{array}{l}\text { Merops philippinus (Linnaeus, } \\
\text { 1766) }\end{array}$ & निलपुछे, मुरली चरा & LC & - & Insectivore & S & OF \\
\hline
\end{tabular}




\begin{tabular}{|c|c|c|c|c|c|c|c|c|}
\hline & $\begin{array}{l}\text { Order/ Family/ Common } \\
\text { name }\end{array}$ & Scientific name & Nepali name & IUCN & CITES & $\begin{array}{l}\text { Foraging } \\
\text { guild }\end{array}$ & $\begin{array}{l}\text { Migration } \\
\text { group }\end{array}$ & $\begin{array}{l}\text { Habitat } \\
\text { Type }\end{array}$ \\
\hline \multirow[t]{3}{*}{121} & $\begin{array}{l}\text { Chestnut-headed Bee- } \\
\text { eater }\end{array}$ & $\begin{array}{l}\text { Merops leschenaulti (Vieillot, } \\
\text { 1817) }\end{array}$ & कटुसे टाउके मुरली चरा & LC & - & Insectivore & $S$ & OF \\
\hline & CUCULIFORMES & & & & & & & \\
\hline & Cuculidae & & & & & & & \\
\hline 122 & Banded Bay Cuckoo & $\begin{array}{l}\text { Cacomantis sonneratii (Latham, } \\
\text { 1790) }\end{array}$ & धर्के खैरो कोइली & LC & - & Insectivore & $\mathrm{R}$ & $\mathrm{FB}$ \\
\hline 123 & Chestnut-winged Cuckoo & $\begin{array}{l}\text { Clamator coromandus (Linnaeus, } \\
1766 \text { ) }\end{array}$ & ठूलो जुरे कोईली & LC & - & Insectivore & $S$ & $\mathrm{FB}$ \\
\hline 124 & Common Cuckoo & Cuculus canorus (Linnaeus, 1758) & कुक्कु & LC & - & Insectivore & S & $\mathrm{FB}$ \\
\hline 125 & Common Hawk-cuckoo & Hierococcyx varius (Vahl, 1797) & भैरब चरो & LC & - & Insectivore & $\mathrm{R}$ & $\mathrm{FB}$ \\
\hline 126 & Greater Coucal & $\begin{array}{l}\text { Centropus sinensis (Stephens, } \\
\text { 1815) }\end{array}$ & ढोढे गोकुल & LC & - & Insectivore & $\mathrm{R}$ & OF \\
\hline 127 & Green-billed Malkoha & $\begin{array}{l}\text { Phaenicophaeus tristis (Lesson, } \\
\text { 1830) }\end{array}$ & हरित माल कौवा & LC & - & Insectivore & $\mathrm{R}$ & $\mathrm{FB}$ \\
\hline 128 & Grey-bellied Cuckoo & $\begin{array}{l}\text { Cacomantis passerinus (Vahl, } \\
\text { 1797) }\end{array}$ & सुत्तरि कोईली & LC & - & Insectivore & $S$ & $\mathrm{FB}$ \\
\hline 129 & Indian Cuckoo & $\begin{array}{l}\text { Cuculus micropterus (Gould, } \\
\text { 1837) }\end{array}$ & काफल पाक्यो & LC & - & Insectivore & $S$ & $\mathrm{FB}$ \\
\hline 130 & Jacobin Cuckoo & $\begin{array}{l}\text { Clamator jacobinus (Boddaert, } \\
1783 \text { ) }\end{array}$ & जुरे कोईली & LC & - & Insectivore & $S$ & $\mathrm{FB}$ \\
\hline 131 & Large Hawk-cuckoo & $\begin{array}{l}\text { Hierococcyx sparverioides (Vigors, } \\
\text { 1832) }\end{array}$ & बिऊ कुईयो कोइली & LC & - & Insectivore & $S$ & $\mathrm{FB}$ \\
\hline 132 & Lesser Coucal & $\begin{array}{l}\text { Centropus bengalensis (Gmelin, } \\
\text { 1788) }\end{array}$ & सानो गोकुल & LC & - & Insectivore & $\mathrm{R}$ & GB \\
\hline 133 & Lesser Cuckoo & $\begin{array}{l}\text { Cuculus poliocephalus (Latham, } \\
1790)\end{array}$ & सानो कोईली & LC & - & Insectivore & S & $\mathrm{FB}$ \\
\hline 134 & Oriental Cuckoo & Cuculus saturatus* (Gould, 1845) & पूर्विय कोईली & LC & - & Insectivore & S & $\mathrm{FB}$ \\
\hline 135 & Sirkeer Malkoha & $\begin{array}{l}\text { Taccocua leschenaultii (Lesson, } \\
\text { 1830) }\end{array}$ & न्याउरी मालकौवा & LC & - & Insectivore & $\mathrm{R}$ & $\mathrm{FB}$ \\
\hline 136 & $\begin{array}{l}\text { Square-tailed Drongo- } \\
\text { cuckoo }\end{array}$ & $\begin{array}{l}\text { Surniculus lugubris (Horsfield, } \\
\text { 1821) }\end{array}$ & चिबे कोईली & LC & - & Insectivore & S & $\mathrm{FB}$ \\
\hline \multirow[t]{3}{*}{137} & Asian Koel & $\begin{array}{l}\text { Eudynamys scolopacea (Linnaeus, } \\
\text { 1758) }\end{array}$ & कोईली & LC & - & Insectivore & $S$ & OF \\
\hline & FALCONIFORMES & & & & & & & \\
\hline & Falconidae & & & & & & & \\
\hline 138 & Collared Falconet & $\begin{array}{l}\text { Microhierax caerulescens } \\
\text { (Linnaeus, 1758) }\end{array}$ & पौरी बाज & LC & II & Insectivore & $\mathrm{R}$ & $\mathrm{FB}$ \\
\hline 139 & Common Kestrel & $\begin{array}{l}\text { Falco tinnunculus (Linnaeus, } \\
1758 \text { ) }\end{array}$ & बौडाई & LC & II & Carnivore & W & OF \\
\hline 140 & Peregrine Falcon & Falco peregrinus (Tunstall, 1771) & शाही बाज & LC & II & Carnivore & W & $\mathrm{FB}$ \\
\hline \multirow[t]{3}{*}{141} & Red- necked Falcon & Falco chicquera (Daudin, 1800) & रातो टाउके बौडाइ & NT & ॥ & Carnivore & $\mathrm{R}$ & $\mathrm{FB}$ \\
\hline & GALLIFORMES & & & & & & & \\
\hline & Phasianidae & & & & & & & \\
\hline 142 & Black Francolin & $\begin{array}{l}\text { Francolinus francolinus (Linnaeus, } \\
1766 \text { ) }\end{array}$ & तीत्रा & LC & - & Granivore & $\mathrm{R}$ & OF \\
\hline 143 & Common Quail & $\begin{array}{l}\text { Coturnix coturnix* (Linnaeus, } \\
1758 \text { ) }\end{array}$ & बट्टाई & LC & - & Granivore & W & OF \\
\hline 144 & Indian Peafowl & Pavo cristatus \# (Linnaeus, 1758) & मयुर & LC & III & Omnivore & R & $\mathrm{FB}$ \\
\hline \multirow[t]{3}{*}{145} & Red junglefowl & Gallus gallus (Linnaeus, 1758) & लुंईचे & LC & - & Omnivore & R & $\mathrm{FB}$ \\
\hline & GRUIFORMES & & & & & & & \\
\hline & Rallidae & & & & & & & \\
\hline 146 & Brown Crake & Zapornia akool (Sykes, 1832) & ध्वाँसे सिम कुखुरा & LC & - & $\begin{array}{c}\text { Aquatic } \\
\text { invertebrates }\end{array}$ & $\mathrm{R}$ & WB \\
\hline 147 & Common Coot & Fulica atra (Linnaeus, 1758) & सेतो थोप्ले सिमकुखुरा & LC & - & $\begin{array}{c}\text { Aquatic } \\
\text { invertebrates }\end{array}$ & W & WB \\
\hline 148 & Common Moorhen & $\begin{array}{l}\text { Gallinula chloropus (Linnaeus, } \\
\text { 1758) }\end{array}$ & बगाले सिमकुखुरा & LC & - & $\begin{array}{c}\text { Aquatic } \\
\text { invertebrates }\end{array}$ & $\mathrm{R}$ & WB \\
\hline 149 & Western Swamphen & $\begin{array}{l}\text { Porphyrio porphyrio (Linnaeus, } \\
\text { 1758) }\end{array}$ & कुर्मा & LC & - & Omnivore & $\mathrm{R}$ & WB \\
\hline 150 & Ruddy-breasted Crake & Zapornia fusca (Linnaeus, 1766) & घोल कस्दरी & LC & - & Omnivore & $\mathrm{R}$ & WB \\
\hline
\end{tabular}




\begin{tabular}{|c|c|c|c|c|c|c|c|c|}
\hline & $\begin{array}{l}\text { Order/ Family/ Common } \\
\text { name }\end{array}$ & Scientific name & Nepali name & IUCN & CITES & $\begin{array}{l}\text { Foraging } \\
\text { guild }\end{array}$ & $\begin{array}{l}\text { Migration } \\
\text { group }\end{array}$ & $\begin{array}{c}\text { Habitat } \\
\text { Type }\end{array}$ \\
\hline 151 & Watercock & Gallicrex cinerea (Gmelin, 1789) & जल कुखुरा & LC & - & Herbivore & $S$ & WB \\
\hline \multirow[t]{3}{*}{152} & $\begin{array}{l}\text { White-breasted } \\
\text { Waterhen }\end{array}$ & $\begin{array}{l}\text { Amaurornis phoenicurus } \\
\text { (Pennant, 1769) }\end{array}$ & सिमकुखुरा & LC & - & $\begin{array}{c}\text { Aquatic } \\
\text { invertebrates }\end{array}$ & $\mathrm{R}$ & WB \\
\hline & PASSERIFORMES & & & & & & & \\
\hline & Acrocephalidae & & & & & & & \\
\hline 153 & Blyth's Reed-warbler & $\begin{array}{l}\text { Acrocephalus dumetorum (Blyth, } \\
\text { 1849) }\end{array}$ & ट्याक्ट्याके & LC & - & Insectivore & w & $\mathrm{FB}$ \\
\hline 154 & Paddyfield Warbler & $\begin{array}{l}\text { Acrocephalus agricola (Jerdon, } \\
1845 \text { ) }\end{array}$ & सानो ट्याक्ट्याके & LC & - & Insectivore & W & OF \\
\hline \multirow[t]{2}{*}{155} & Thick-billed Warbler & Arundinax aedon (Pallas, 1776) & मोटोठुँडे ट्याक्टयाके & LC & - & Insectivore & W & OF \\
\hline & Aegithinidae & & & & & & & \\
\hline \multirow[t]{2}{*}{156} & Common lora & Aegithina tiphia (Linnaeus, 1758) & सुसेली चरी & LC & - & Insectivore & $\mathrm{R}$ & FB \\
\hline & Alaudidae & & & & & & & \\
\hline 157 & Bengal Bushlark & $\begin{array}{l}\text { Mirafra assamica (Horsfield, } \\
\text { 1840) }\end{array}$ & भार्दवाज & LC & - & Insectivore & $\mathrm{R}$ & OF \\
\hline 158 & Oriental Skylark & Alauda gulgula\# (Franklin, 1831) & ब्रहमीचटी भार्दवाज & LC & - & Insectivore & $\mathrm{R}$ & OF \\
\hline \multirow[t]{2}{*}{159} & Sand Lark & Alaudala raytal* (Blyth, 1845) & बगर भार्दवाज & LC & - & Insectivore & $\mathrm{R}$ & OF \\
\hline & Artamidae & & & & & & & \\
\hline \multirow[t]{2}{*}{160} & Ashy Woodswallow & Artamus fuscus (Vieillot, 1817) & मिथुन & LC & - & Insectivore & $\mathrm{R}$ & OF \\
\hline & Campephagidae & & & & & & & \\
\hline 161 & $\begin{array}{l}\text { Black-headed } \\
\text { Cuckooshrike }\end{array}$ & $\begin{array}{l}\text { Lalage melanoptera\# (Rüppell, } \\
\text { 1839) }\end{array}$ & कालो टाउके बिरहि चरा & LC & - & Insectivore & $\mathrm{R}$ & $\mathrm{FB}$ \\
\hline 162 & $\begin{array}{l}\text { Black-winged } \\
\text { Cuckooshrike }\end{array}$ & $\begin{array}{l}\text { Lalage melaschistos\# (Hodgson, } \\
\text { 1836) }\end{array}$ & कालो बिरहि चरा & LC & - & Insectivore & $\mathrm{R}$ & $\mathrm{FB}$ \\
\hline 163 & Indian Cuckooshrike & Coracina macei (Lesson, 1830) & लठसक् बिरहि चरा & LC & - & Insectivore & $\mathrm{R}$ & $\mathrm{FB}$ \\
\hline 164 & Rosy Minivet & $\begin{array}{l}\text { Pericrocotus roseus (Vieillot, } \\
\text { 1818) }\end{array}$ & गुलाफी रानीचरा & LC & - & Insectivore & $\mathrm{R}$ & $\mathrm{FB}$ \\
\hline 165 & Scarlet Minivet & $\begin{array}{l}\text { Pericrocotus flammeus (Latham, } \\
\text { 1790) }\end{array}$ & रानीचरा & LC & - & Insectivore & $\mathrm{R}$ & $\mathrm{FB}$ \\
\hline \multirow[t]{2}{*}{166} & Small Minivet & $\begin{array}{l}\text { Pericrocotus cinnamomeus } \\
\text { (Linnaeus, 1766) }\end{array}$ & सानो रानीचरा & LC & - & Insectivore & $\mathrm{R}$ & $\mathrm{FB}$ \\
\hline & Cettiidae & & & & & & & \\
\hline \multirow[t]{2}{*}{167} & Aberrant Bush-warbler & $\begin{array}{l}\text { Horornis flavolivaceus \# (Blyth, } \\
\text { 1845) }\end{array}$ & पित हरितभाडी फिस्टो & LC & - & Insectivore & $\mathrm{R}$ & $\mathrm{FB}$ \\
\hline & Chloropseidae & & & & & & & \\
\hline \multirow[t]{2}{*}{168} & Golden-fronted Leafbird & $\begin{array}{l}\text { Chloropsis aurifrons (Temminck, } \\
\text { 1829) }\end{array}$ & कृण्ण कण्ठे हरितचरी & LC & - & Insectivore & $\mathrm{R}$ & FB \\
\hline & Cisticolidae & & & & & & & \\
\hline 169 & Ashy Prinia & Prinia socialis (Sykes, 1832) & टुनुक् घाँसेफिस्टो & LC & - & Insectivore & $\mathrm{R}$ & GB \\
\hline 170 & Common Tailorbird & $\begin{array}{l}\text { Orthotomus sutorius (Pennant, } \\
1769 \text { ) }\end{array}$ & सोलिफिस्टो & LC & - & Insectivore & $\mathrm{R}$ & FB \\
\hline 171 & Grey-breasted Prinia & Prinia hodgsonii (Blyth, 1844) & फुस्रोछाति घाँसेफिस्टो & LC & - & Insectivore & $\mathrm{R}$ & $\mathrm{FB}$ \\
\hline 172 & Grey-crowned Prinia & $\begin{array}{l}\text { Prinia cinereocapilla* (Moore, } \\
\text { 1854) }\end{array}$ & घेघरी घाँसेफिस्टो & VU & - & Insectivore & $\mathrm{R}$ & GB \\
\hline 173 & Jungle Prinia & Prinia sylvatica (Jerdon, 1840) & जड़ल घाँसेफिस्टो & LC & - & Insectivore & $\mathrm{R}$ & GB \\
\hline 174 & Plain Prinia & Prinia inornata (Sykes, 1832) & काक्री घाँसेफिस्टो & LC & - & Insectivore & $\mathrm{R}$ & GB \\
\hline 175 & Yellow-bellied Prinia & $\begin{array}{l}\text { Prinia flaviventris (Delessert, } \\
\text { 1840) }\end{array}$ & पिठोदर घाँसेफिस्टो & LC & - & Insectivore & $\mathrm{R}$ & GB \\
\hline \multirow[t]{2}{*}{176} & Zitting Cisticola & $\begin{array}{l}\text { Cisticola juncidis (Rafinesque, } \\
\text { 1810) }\end{array}$ & सिरु फिस्टो & LC & - & Insectivore & $\mathrm{R}$ & GB \\
\hline & Corvidae & & & & & & & \\
\hline 177 & Grey treepie & $\begin{array}{l}\text { Dendrocitta formosae @ (R. } \\
\text { Swinhoe, 1863) }\end{array}$ & पहाडी कोकले & LC & - & Frugivore & $\mathrm{R}$ & $\mathrm{FB}$ \\
\hline 178 & House Crow & Corvus splendens (Vieillot, 1817) & कण्ठे काग & LC & - & Omnivore & $\mathrm{R}$ & OF \\
\hline 179 & Large-billed Crow & $\begin{array}{l}\text { Corvus macrorhynchos (Wagler, } \\
\text { 1827) }\end{array}$ & कालो कौवा & LC & - & Omnivore & $\mathrm{R}$ & OF \\
\hline 180 & Rufous Treepie & $\begin{array}{l}\text { Dendrocitta vagabunda (Latham, } \\
\text { 1790) }\end{array}$ & कोकले & LC & - & Frugivore & $\mathrm{R}$ & $\mathrm{FB}$ \\
\hline
\end{tabular}




\begin{tabular}{|c|c|c|c|c|c|c|c|c|}
\hline & $\begin{array}{l}\text { Order/ Family/ Common } \\
\text { name }\end{array}$ & Scientific name & Nepali name & IUCN & CITES & $\begin{array}{l}\text { Foraging } \\
\text { guild }\end{array}$ & $\begin{array}{l}\text { Migration } \\
\text { group }\end{array}$ & $\begin{array}{c}\text { Habitat } \\
\text { Type }\end{array}$ \\
\hline & Dicaeidae & & & & & & & \\
\hline 181 & Pale-billed Flowerpecker & $\begin{array}{l}\text { Dicaeum erythrorhynchos \# } \\
\text { (Latham, 1790) }\end{array}$ & रातोठुंडे पुष्पकोकिल & LC & - & Frugivore & $\mathrm{R}$ & $\mathrm{FB}$ \\
\hline 182 & Plain Flowerpecker & $\begin{array}{l}\text { Dicaeum minullum (R. Swinhoe, } \\
\text { 1870) }\end{array}$ & समरुप पुष्पकोकिल & LC & - & Frugivore & $\mathrm{R}$ & $\mathrm{FB}$ \\
\hline 183 & $\begin{array}{l}\text { Thick-billed } \\
\text { Flowerpecker }\end{array}$ & Dicaeum agile@ (Tickell, 1833) & मोटोठुंडे पुष्पकोकिल & LC & - & Frugivore & $\mathrm{R}$ & $\mathrm{FB}$ \\
\hline \multirow[t]{2}{*}{184} & $\begin{array}{l}\text { Yellow-vented } \\
\text { Flowerpecker }\end{array}$ & $\begin{array}{l}\text { Dicaeum chrysorrheum@ } \\
\text { (Temminck, 1829) }\end{array}$ & पितनिर्गम पुष्पकोकिल & LC & - & Frugivore & $\mathrm{R}$ & $\mathrm{FB}$ \\
\hline & Dicruridae & & & & & & & \\
\hline 185 & Ashy Drongo & $\begin{array}{l}\text { Dicrurus leucophaeus (Vieillot, } \\
\text { 1817) }\end{array}$ & ध्वाँसे चिबे & LC & - & Insectivore & $\mathrm{s}$ & $\mathrm{FB}$ \\
\hline 186 & Black Drongo & $\begin{array}{l}\text { Dicrurus macrocercus (Vieillot, } \\
\text { 1817) }\end{array}$ & चिबे & LC & - & Insectivore & $\mathrm{R}$ & OF \\
\hline 187 & Bronzed Drongo & Dicrurus aeneus (Vieillot, 1817) & आकाशे चिबे & LC & - & Insectivore & $\mathrm{R}$ & FB \\
\hline 188 & Crow-billed Drongo & $\begin{array}{l}\text { Dicrurus annectans (Hodgson, } \\
\text { 1836) }\end{array}$ & कागठुँड चिबे & LC & - & Insectivore & $\mathrm{s}$ & $\mathrm{FB}$ \\
\hline 189 & $\begin{array}{l}\text { Greater Racket-tailed } \\
\text { Drongo }\end{array}$ & $\begin{array}{l}\text { Dicrurus paradiseus (Linnaeus, } \\
1766 \text { ) }\end{array}$ & भीमराज चिबे & LC & - & Insectivore & $\mathrm{R}$ & $\mathrm{FB}$ \\
\hline 190 & Hair-crested Drongo & $\begin{array}{l}\text { Dicrurus hottentottus (Linnaeus, } \\
\text { 1766) }\end{array}$ & केसराज चिबे & LC & - & Insectivore & $\mathrm{R}$ & $\mathrm{FB}$ \\
\hline 191 & $\begin{array}{l}\text { Lesser Racket-tailed } \\
\text { Drongo }\end{array}$ & $\begin{array}{l}\text { Dicrurus remifer (Temminck, } \\
\text { 1823) }\end{array}$ & भृड़राज चिबे & LC & - & Insectivore & $\mathrm{R}$ & $\mathrm{FB}$ \\
\hline \multirow[t]{2}{*}{192} & White-bellied Drongo & $\begin{array}{l}\text { Dicrurus caerulescens* (Linnaeus, } \\
1758 \text { ) }\end{array}$ & सेतोपेटे चिबे & LC & - & Insectivore & $\mathrm{R}$ & $\mathrm{FB}$ \\
\hline & Emberizidae & & & & & & & \\
\hline 193 & Crested Bunting & Emberiza lathami (Gray, 1831) & जुरे बगेडी & LC & - & Granivore & $\mathrm{R}$ & OF \\
\hline \multirow[t]{2}{*}{194} & Yellow-breasted Bunting & Emberiza aureola (Pallas, 1773) & बगाले बगेडी & CR & - & Granivore & w & OF \\
\hline & Estrildidae & & & & & & & \\
\hline 195 & Red Avadavat & $\begin{array}{l}\text { Amandava amandava (Linnaeus, } \\
\text { 1758) }\end{array}$ & रातो मुनिया & LC & - & Granivore & $\mathrm{R}$ & GB \\
\hline 196 & Scaly-breasted Munia & $\begin{array}{l}\text { Lonchura punctulata (Linnaeus, } \\
1758 \text { ) }\end{array}$ & कोटेरो मुनिया & LC & - & Granivore & $\mathrm{R}$ & OF \\
\hline 197 & Tricoloured Munia & $\begin{array}{l}\text { Lonchura malacca (Linnaeus, } \\
\text { 1766) }\end{array}$ & कालेटाउके मुनिया & LC & - & Granivore & $\mathrm{S}$ & GB \\
\hline \multirow[t]{2}{*}{198} & White-rumped Munia & $\begin{array}{l}\text { Lonchura striata@ (Linnaeus, } \\
\text { 1766) }\end{array}$ & सेतो ढाढे मुनिया & LC & - & Granivore & $\mathrm{R}$ & GB \\
\hline & Eurylaimidae & & & & & & & \\
\hline \multirow[t]{2}{*}{199} & Long-tailed Broadbill & $\begin{array}{l}\text { Psarisomus dalhousiae } \$ \\
\text { (Jameson, 1835) }\end{array}$ & चित्रकुट & LC & - & Insectivore & $\mathrm{R}$ & $\mathrm{FB}$ \\
\hline & Fringillidae & & & & & & & \\
\hline \multirow[t]{2}{*}{200} & Common Rosefinch & $\begin{array}{l}\text { Carpodacus erythrinus (Pallas, } \\
1770 \text { ) }\end{array}$ & अमोगा तितु & LC & - & Granivore & W & $\mathrm{FB}$ \\
\hline & Hirundinidae & & & & & & & \\
\hline 201 & Asian Plain Martin & Riparia chinensis (J. E. Gray, 1830) & भित्तेगौथली & LC & - & Insectivore & $\mathrm{R}$ & OF \\
\hline 202 & Barn Swallow & Hirundo rustica (Linnaeus, 1758) & घर गौंथली & LC & - & Insectivore & $\mathrm{R}$ & OF \\
\hline 203 & Sand Martin & Riparia riparia (Linnaeus, 1758) & गलहरी भित्ते गौथली & LC & - & Insectivore & $\mathrm{R}$ & OF \\
\hline 204 & Nepal House Martin & $\begin{array}{l}\text { Delichon nipalense \$ (Moore, } \\
\text { 1854) }\end{array}$ & नेपाल भिर गौंथली & LC & - & Insectivore & $\mathrm{R}$ & OF \\
\hline \multirow[t]{2}{*}{205} & Red-rumped Swallow & $\begin{array}{l}\text { Cecropis daurica\# (Laxmann, } \\
\text { 1769) }\end{array}$ & गेरूकाटी गौथथली & LC & - & Insectivore & $\mathrm{R}$ & OF \\
\hline & Laniidae & & & & & & & \\
\hline 206 & Brown Shrike & Lanius cristatus (Linnaeus, 1758) & खैरो भद्राई & LC & - & Insectivore & W & OF \\
\hline 207 & Grey-backed Shrike & Lanius tephronotus (Vigors, 1831) & हिमाली भद्राई & LC & - & Insectivore & W & OF \\
\hline \multirow[t]{2}{*}{208} & Long-tailed Shrike & Lanius schach (Linnaeus, 1758) & भद्राई & LC & - & Insectivore & $\mathrm{R}$ & OF \\
\hline & Leiotrichidae & & & & & & & \\
\hline 209 & Common babbler & Argya caudata\$s(Dumont, 1823) & कुरुबाहुक् भ्याकुर & LC & - & Insectivore & $\mathrm{R}$ & GB \\
\hline 210 & Jungle Babbler & $\begin{array}{l}\text { Turdoides striata\# (Dumont, } \\
\text { 1823) }\end{array}$ & बगाले भ्याकुर & LC & - & Insectivore & $\mathrm{R}$ & $\mathrm{FB}$ \\
\hline
\end{tabular}




\begin{tabular}{|c|c|c|c|c|c|c|c|c|}
\hline & $\begin{array}{l}\text { Order/ Family/ Common } \\
\text { name }\end{array}$ & Scientific name & Nepali name & IUCN & CITES & $\begin{array}{l}\text { Foraging } \\
\text { guild }\end{array}$ & $\begin{array}{l}\text { Migration } \\
\text { group }\end{array}$ & $\begin{array}{c}\text { Habitat } \\
\text { Type }\end{array}$ \\
\hline \multirow[t]{2}{*}{211} & Striated Babbler & Argya earlei@ (Blyth, 1844) & खर भ्याकुर & LC & - & Insectivore & $\mathrm{R}$ & $\mathrm{GB}$ \\
\hline & Locustellidae & & & & & & & \\
\hline 212 & Bristled Grassbird & Chaetornis striata\# (Jerdon, 1841) & चिप्लिक घाँसे चरी & VU & - & Insectivore & $\mathrm{R}$ & GB \\
\hline \multirow[t]{2}{*}{213} & Spotted Bush Warbler & $\begin{array}{l}\text { Locustella thoracica* (Blyth, } \\
1845 \text { ) }\end{array}$ & थोप्ले काडीफिस्टो & LC & - & Insectivore & $\mathrm{R}$ & GB \\
\hline & Monarchidae & & & & & & & \\
\hline \multirow[t]{2}{*}{214} & Black-naped Monarch & $\begin{array}{l}\text { Hypothymis azurea (Boddaert, } \\
\text { 1783) }\end{array}$ & कालो गर्धन राजचरी & LC & - & Insectivore & $\mathrm{R}$ & $\mathrm{FB}$ \\
\hline & Motacillidae & & & & & & & \\
\hline 215 & Citrine Wagtail & Motacilla citreola (Pallas, 1776) & बेसारे टिक्टिके & LC & - & Insectivore & W & PW \\
\hline 216 & Forest Wagtail & $\begin{array}{l}\text { Dendronanthus indicus (Gmelin, } \\
\text { 1789) }\end{array}$ & वन टिक्टिके & LC & - & Insectivore & $P$ & $\mathrm{FB}$ \\
\hline 217 & Grey Wagtail & Motacilla cinerea (Tunstall, 1771) & फुस्रो टिक्टिके & LC & - & Insectivore & W & PW \\
\hline 218 & Olive-backed Pipit & $\begin{array}{l}\text { Anthus hodgsoni (Richmond, } \\
\text { 1907) }\end{array}$ & रुख चुईंया & LC & - & Insectivore & W & $\mathrm{FB}$ \\
\hline 219 & Paddyfield Pipit & Anthus rufulus (Vieillot, 1818) & आलि चुइंया & LC & - & Insectivore & $\mathrm{R}$ & OF \\
\hline 220 & Richard's Pipit & Anthus richardi (Vieillot, 1818) & लामाऔंले चुईंया & LC & - & Insectivore & W & OF \\
\hline 221 & Rosy Pipit & Anthus roseatus (Blyth, 1847) & गुलाफी कण्ठे चुईया & LC & - & Insectivore & W & PW \\
\hline 222 & Tawny Pipit & $\begin{array}{l}\text { Anthus campestris (Linnaeus, } \\
1758 \text { ) }\end{array}$ & ढुलिका चुईंया & LC & - & Insectivore & W & OF \\
\hline 223 & Western Yellow Wagtail & Motacilla flava* (Linnaeus, 1758) & पहेंलो टिक्टिके & LC & - & Insectivore & W & PW \\
\hline 224 & White Wagtail & Motacilla alba (Linnaeus, 1758) & सेतो टिक्टिके & LC & - & Insectivore & W & OF \\
\hline \multirow[t]{2}{*}{225} & White-browed Wagtail & $\begin{array}{l}\text { Motacilla maderaspatensis } \\
\text { (Gmelin, 1789) }\end{array}$ & खोले टिक्टिके & LC & - & Insectivore & $\mathrm{R}$ & PW \\
\hline & Muscicapidae & & & & & & & \\
\hline 226 & Asian Brown Flycatcher & Muscicapa dauurica (Pallas, 1811) & धुसर अर्जुनक & LC & - & Insectivore & S & $\mathrm{FB}$ \\
\hline 227 & Black Redstart & $\begin{array}{l}\text { Phoenicurus ochruros (S. G. } \\
\text { Gmelin, 1774) }\end{array}$ & ध्याप्ची खঙ्जरी & LC & - & Insectivore & W & OF \\
\hline 228 & Black-backed Forktail & $\begin{array}{l}\text { Enicurus immaculatus (Hodgson, } \\
\text { 1836) }\end{array}$ & कालोढाडे खोलेधोबिनी & LC & - & Insectivore & $\mathrm{R}$ & PW \\
\hline 229 & Blue Rock-thrush & $\begin{array}{l}\text { Monticola solitarius (Linnaeus, } \\
1758 \text { ) }\end{array}$ & उमा चाँचर & LC & - & Insectivore & $\mathrm{R}$ & $\mathrm{FB}$ \\
\hline 230 & Blue Whistling-thrush & $\begin{array}{l}\text { Myophonus caeruleus (Scopoli, } \\
\text { 1786) }\end{array}$ & कलचौडे & LC & - & Omnivore & $\mathrm{R}$ & $\mathrm{FB}$ \\
\hline 231 & Bluethroat & $\begin{array}{l}\text { Cyanecula svecica (Linnaeus, } \\
1758 \text { ) }\end{array}$ & भुमिचर निलकण्ठ & LC & - & Insectivore & W & $\mathrm{FB}$ \\
\hline 232 & Common Stonechat & $\begin{array}{l}\text { Saxicola torquatus (Linnaeus, } \\
\text { 1766) }\end{array}$ & भेकभेक भ्याप्सी & LC & - & Insectivore & W & GB \\
\hline 233 & Dark-sided Flycatcher & Muscicapa sibirica (Gmelin, 1789) & ध्वाँसे अर्जुनक & LC & - & Insectivore & W & FB \\
\hline 234 & Grey Bushchat & $\begin{array}{l}\text { Saxicola ferreus (Gray \& Gray, } \\
\text { 1847) }\end{array}$ & हिमाली कयाप्सी & LC & - & Insectivore & W & OF \\
\hline 235 & Himalayan Rubythroat & Calliope pectoralis\# (Gould, 1837) & साईबेरियन रातोकण्ठ & LC & - & Insectivore & W & $\mathrm{FB}$ \\
\hline 236 & Little Forktail & Enicurus scouleri (Vigors, 1832) & गंगा खोलेधोबिनी & LC & - & Insectivore & $\mathrm{R}$ & PW \\
\hline 237 & Oriental Magpie-robin & $\begin{array}{l}\text { Copsychus saularis (Linnaeus, } \\
1758 \text { ) }\end{array}$ & धोबी चरा & LC & - & Insectivore & $\mathrm{R}$ & OF \\
\hline 238 & Pale-Chinned Flycatcher & Cyornis poliogenys (Brooks, 1879) & नौनि कण्ठे अर्जुनक & LC & - & Insectivore & $\mathrm{R}$ & $\mathrm{FB}$ \\
\hline 239 & Pied Bush Chat & Saxicola caprata (Linnaeus, 1766) & काले कयाप्सी & LC & - & Insectivore & $\mathrm{R}$ & GB \\
\hline 240 & $\begin{array}{l}\text { Plumbeous Water } \\
\text { Redstart }\end{array}$ & $\begin{array}{l}\text { Phoenicurus fuliginosus (Vigors, } \\
\text { 1831) }\end{array}$ & निलाम्बर जलखः्जरी & LC & - & $\begin{array}{c}\text { Aquatic } \\
\text { invertebrates }\end{array}$ & W & PW \\
\hline 241 & Pygmy Blue-flycatcher & Ficedula hodgsoni (Moore, 1854) & नीलढाडे अर्जुनक & LC & - & Insectivore & W & $\mathrm{FB}$ \\
\hline 242 & Red-breasted Flycatcher & Ficedula parva* (Bechstein, 1792) & लालबक्स अर्जुनक & LC & - & Insectivore & W & $\mathrm{FB}$ \\
\hline 243 & Red-throated Flycatcher & Ficedula albicilla (Pallas, 1811) & रातो कण्ठे अर्जुनक & LC & - & Insectivore & W & $\mathrm{FB}$ \\
\hline 244 & Rufous-bellied Niltava & $\begin{array}{l}\text { Niltava sundara@ (Hodgson, } \\
\text { 1837) }\end{array}$ & सुन्दर निलताभा & LC & - & Insectivore & $\mathrm{R}$ & $\mathrm{FB}$ \\
\hline 245 & Rusty-tailed Flycatcher & $\begin{array}{l}\text { Ficedula ruficauda (Swainson, } \\
\text { 1838) }\end{array}$ & धुसार अर्जुनक & LC & - & Insectivore & $S$ & $\mathrm{FB}$ \\
\hline 246 & Siberian Rubythroat & Calliope calliope (Pallas, 1776) & हिमाली रातोकण्ठ & LC & - & Insectivore & W & $\mathrm{FB}$ \\
\hline
\end{tabular}




\begin{tabular}{|c|c|c|c|c|c|c|c|c|}
\hline & $\begin{array}{l}\text { Order/ Family/ Common } \\
\text { name }\end{array}$ & Scientific name & Nepali name & IUCN & CITES & $\begin{array}{l}\text { Foraging } \\
\text { guild }\end{array}$ & $\begin{array}{l}\text { Migration } \\
\text { group }\end{array}$ & $\begin{array}{c}\text { Habitat } \\
\text { Type }\end{array}$ \\
\hline 247 & Slaty-backed Forktail & $\begin{array}{l}\text { Enicurus schistaceus (Hodgson, } \\
1836 \text { ) }\end{array}$ & फुस्रोढाडे खोलेधोबिनी & LC & - & Insectivore & $\mathrm{R}$ & PW \\
\hline 248 & Slaty-blue Flycatcher & $\begin{array}{l}\text { Ficedula tricolor@ (Hodgson, } \\
\text { 1845) }\end{array}$ & टिक्टिके अर्जुनक & LC & - & Insectivore & $\mathrm{R}$ & $\mathrm{FB}$ \\
\hline 249 & Ultramarine Flycatcher & $\begin{array}{l}\text { Ficedula superciliaris \$ (Jerdon, } \\
1840 \text { ) }\end{array}$ & निलश्वेत अर्जुनक & LC & - & Insectivore & $\mathrm{R}$ & $\mathrm{FB}$ \\
\hline 250 & Verditer Flycatcher & $\begin{array}{l}\text { Eumyias thalassina (Swainson, } \\
\text { 1838) }\end{array}$ & निलतुथो अर्जुनक & LC & - & Insectivore & W & $\mathrm{FB}$ \\
\hline 251 & $\begin{array}{l}\text { White-browed Bush } \\
\text { Robin }\end{array}$ & Tarsigerindicus@ (Vieillot, 1817) & सेतो आँखीभडँ रविन & LC & - & Insectivore & $\mathrm{R}$ & $\mathrm{FB}$ \\
\hline 252 & $\begin{array}{l}\text { White-capped Water } \\
\text { Redstart }\end{array}$ & $\begin{array}{l}\text { Phoenicurus leucocephalus\# } \\
\text { (Vigors, 1831) }\end{array}$ & सेतोटाउके जलखः्जरी & LC & - & $\begin{array}{c}\text { Aquatic } \\
\text { invertebrates }\end{array}$ & $\mathrm{R}$ & PW \\
\hline 253 & White-rumped Shama & $\begin{array}{l}\text { Kittacincla malabarica\# (Scopoli, } \\
\text { 1788) }\end{array}$ & श्यामा & LC & - & Insectivore & $\mathrm{R}$ & $\mathrm{FB}$ \\
\hline 254 & White-tailed Blue Robin & $\begin{array}{l}\text { Myiomela leucura (Hodgson, } \\
\text { 1845) }\end{array}$ & सेतोपुच्छे रबिन & LC & - & Insectivore & $\mathrm{R}$ & $\mathrm{FB}$ \\
\hline \multirow[t]{2}{*}{255} & White-tailed Stonechat & Saxicola leucurus\# (Blyth, 1847) & काँसे कयापसी & LC & - & Insectivore & $\mathrm{R}$ & GB \\
\hline & Nectariniidae & & & & & & & \\
\hline 256 & Crimson Sunbird & $\begin{array}{l}\text { Aethopyga siparaja (Raffles, } \\
\text { 1822) }\end{array}$ & सिपचराबुड़ेचरा & LC & - & Nectarivore & $\mathrm{R}$ & $\mathrm{FB}$ \\
\hline \multirow[t]{2}{*}{257} & Purple Sunbird & $\begin{array}{l}\text { Cinnyris asiaticus\# (Latham, } \\
\text { 1790) }\end{array}$ & कालो बुड़ेचरा & LC & - & Nectarivore & $\mathrm{R}$ & FB \\
\hline & Oriolidae & & & & & & & \\
\hline 258 & Black-hooded Oriole & $\begin{array}{l}\text { Oriolus xanthornus (Linnaeus, } \\
\text { 1758) }\end{array}$ & कालो टाउके सुनचरी & LC & - & Omnivore & $\mathrm{R}$ & $\mathrm{FB}$ \\
\hline \multirow[t]{2}{*}{259} & Eurasian Golden Oriole & Oriolus oriolus (Linnaeus, 1758) & गाजले सुनचरी & LC & - & Omnivore & S & FB \\
\hline & Paridae & & & & & & & \\
\hline \multirow[t]{2}{*}{260} & Great Tit & Parus major (Linnaeus, 1758) & चिचिल्कोटे & LC & - & Insectivore & $\mathrm{R}$ & $\mathrm{FB}$ \\
\hline & Passeridae & & & & & & & \\
\hline 261 & Yellow-throated Sparrow & $\begin{array}{l}\text { Gymnoris xanthocollis\# (Burton, } \\
\text { 1838) }\end{array}$ & पितकण्ठे भंगेरा & LC & - & Granivore & $\mathrm{R}$ & $\mathrm{FB}$ \\
\hline 262 & Eurasian Tree Sparrow & $\begin{array}{l}\text { Passer montanus (Linnaeus, } \\
1758 \text { ) }\end{array}$ & रूख भँगेरा & LC & - & Granivore & $\mathrm{R}$ & OF \\
\hline \multirow[t]{2}{*}{263} & House Sparrow & $\begin{array}{l}\text { Passer domesticus (Linnaeus, } \\
\text { 1758) }\end{array}$ & भँगेरा & LC & - & Granivore & $\mathrm{R}$ & OF \\
\hline & Pellorneidae & & & & & & & \\
\hline 264 & Indian Grassbird & $\begin{array}{l}\text { Graminicola bengalensis (Jerdon, } \\
\text { 1863) }\end{array}$ & घाँसे चरी & NT & - & Insectivore & $\mathrm{R}$ & GB \\
\hline \multirow[t]{2}{*}{265} & Puff-throated Babbler & $\begin{array}{l}\text { Pellorneum ruficeps (Swainson, } \\
\text { 1832) }\end{array}$ & थोप्ले भ्याँकुर & LC & - & Insectivore & $\mathrm{R}$ & FB \\
\hline & Phylloscopidae & & & & & & & \\
\hline 266 & Blyth's Leaf-warbler & $\begin{array}{l}\text { Phylloscopus reguloides (Blyth, } \\
\text { 1842) }\end{array}$ & तालुधर्के फिस्टो & LC & - & Insectivore & W & $\mathrm{FB}$ \\
\hline 267 & $\begin{array}{l}\text { Chestnut-crowned } \\
\text { Warbler }\end{array}$ & $\begin{array}{l}\text { Phylloscopus castaniceps } \\
\text { (Hodgson, 1845) }\end{array}$ & रातो टाउके फिस्टो & LC & - & Insectivore & $\mathrm{R}$ & $\mathrm{FB}$ \\
\hline 268 & Common Chiffchaff & $\begin{array}{l}\text { Phylloscopus collybita (Vieillot, } \\
\text { 1817) }\end{array}$ & चिच्चिप फिस्टो & LC & - & Insectivore & W & FB \\
\hline 269 & Dusky Warbler & $\begin{array}{l}\text { Phylloscopus fuscatus (Blyth, } \\
\text { 1842) }\end{array}$ & गोधूली फिस्टो & LC & - & Insectivore & W & FB \\
\hline 270 & Green-crowned Warbler & $\begin{array}{l}\text { Phylloscopus burkii\# (Burton, } \\
\text { 1836) [ }\end{array}$ & सुनचस्मे फिस्टो & LC & - & Insectivore & W & FB \\
\hline 271 & Greenish Warbler & $\begin{array}{l}\text { Phylloscopus trochiloides } \\
\text { (Sundevall, 1837) }\end{array}$ & जिभल फिस्टो & LC & - & Insectivore & W & $\mathrm{FB}$ \\
\hline 272 & Grey-hooded Warbler & $\begin{array}{l}\text { Phylloscopus xanthoschistos\# } \\
\text { (Gray \& Gray, 1846) }\end{array}$ & तुमुलकारी फिस्टो & LC & - & Insectivore & W & $\mathrm{FB}$ \\
\hline 273 & Hume's Leaf-warbler & $\begin{array}{l}\text { Phylloscopus humei (Brooks, } \\
\text { 1878) }\end{array}$ & चन्चले फिस्टो & LC & - & Insectivore & $\mathrm{R}$ & FB \\
\hline 274 & Large-billed Leaf-warbler & $\begin{array}{l}\text { Phylloscopus magnirostris(Blyth, } \\
\text { 1843) }\end{array}$ & ठूलोठुँडे फिस्टो & LC & - & Insectivore & W & $\mathrm{FB}$ \\
\hline 275 & Smoky Warbler & $\begin{array}{l}\text { Phylloscopus fuligiventer } \\
\text { (Hodgson, 1845) }\end{array}$ & ध्वाँसे फिस्टो & LC & - & Insectivore & W & GB \\
\hline 276 & Sulphur-bellied Warbler & $\begin{array}{l}\text { Phylloscopus griseolus (Blyth, } \\
\text { 1847) }\end{array}$ & पीतोदर ढुड़ेफिस्टो & LC & - & Insectivore & $S$ & $\mathrm{FB}$ \\
\hline
\end{tabular}




\begin{tabular}{|c|c|c|c|c|c|c|c|c|}
\hline & $\begin{array}{l}\text { Order/ Family/ Common } \\
\text { name }\end{array}$ & Scientific name & Nepali name & IUCN & CITES & $\begin{array}{l}\text { Foraging } \\
\text { guild }\end{array}$ & $\begin{array}{l}\text { Migration } \\
\text { group }\end{array}$ & $\begin{array}{l}\text { Habitat } \\
\text { Type }\end{array}$ \\
\hline 277 & Tickell's Leaf-warbler & Phylloscopus affinis (Tickell, 1833) & पीतोदर फिस्टो & LC & - & Insectivore & W & $\mathrm{FB}$ \\
\hline 278 & $\begin{array}{l}\text { Western Crowned } \\
\text { Warbler }\end{array}$ & $\begin{array}{l}\text { Phylloscopus occipitalis* (Blyth, } \\
\text { 1845) }\end{array}$ & ठूलो तालुधर्के फिस्टो & LC & - & Insectivore & W & $\mathrm{FB}$ \\
\hline \multirow[t]{2}{*}{279} & Whistler's Warbler & $\begin{array}{l}\text { Phylloscopus whistleri\# } \\
\text { (Ticehurst, 1925) }\end{array}$ & सुसेली फिस्टो & LC & - & Insectivore & $\mathrm{R}$ & FB \\
\hline & Pittidae & & & & & & & \\
\hline 280 & Indian Pitta & Pitta brachyura (Linnaeus, 1766) & गाजले पिट्टा & LC & - & Insectivore & S & $\mathrm{FB}$ \\
\hline \multirow[t]{2}{*}{281} & Hooded Pitta & $\begin{array}{l}\text { Pitta sordidida (Statius Müller, } \\
\text { 1776) }\end{array}$ & चित्रक पिट्टा & LC & - & Insectivore & $S$ & $\mathrm{FB}$ \\
\hline & Ploceidae & & & & & & & \\
\hline \multirow[t]{2}{*}{282} & Baya Weaver & $\begin{array}{l}\text { Ploceus philippinus (Linnaeus, } \\
\text { 1766) }\end{array}$ & तोपचरा & LC & - & Granivore & $\mathrm{R}$ & GB \\
\hline & Pnoepygidae & & & & & & & \\
\hline \multirow[t]{2}{*}{283} & Nepal Cupwing & $\begin{array}{l}\text { Pnoepyga immaculata (Martens } \\
\text { \& Eck, 1991) }\end{array}$ & नेपाल ढिकुरेभ्याकुर & LC & - & Insectivore & $\mathrm{R}$ & $\mathrm{FB}$ \\
\hline & Pycnonotidae & & & & & & & \\
\hline 284 & Black Bulbul & $\begin{array}{l}\text { Hypsipetes leucocephalus } \\
\text { (Gmelin, 1789) }\end{array}$ & बाखे जुरेली & LC & - & Omnivore & $\mathrm{R}$ & $\mathrm{FB}$ \\
\hline 285 & Black-capped Bulbul & $\begin{array}{l}\text { Pycnonotus melanicterus } \\
\text { (Gmelin, 1789) }\end{array}$ & कालोकल्की पहेंलो जुरेली & LC & - & Omnivore & $\mathrm{R}$ & $\mathrm{FB}$ \\
\hline 286 & Himalayan Bulbul & $\begin{array}{l}\text { Pycnonotus leucogenys (Gray, } \\
\text { JE, 1835) }\end{array}$ & तार्के जुरेली & LC & - & Omnivore & $\mathrm{R}$ & $\mathrm{FB}$ \\
\hline 287 & Red-vented Bulbul & $\begin{array}{l}\text { Pycnonotus cafer (Linnaeus, } \\
1766 \text { ) }\end{array}$ & जुरेली & LC & - & Omnivore & $\mathrm{R}$ & $\mathrm{FB}$ \\
\hline \multirow[t]{2}{*}{288} & Red-whiskered Bulbul & $\begin{array}{l}\text { Pycnonotus jocosus (Linnaeus, } \\
1758 \text { ) }\end{array}$ & श्वेतभक्ष जुरेली & LC & - & Omnivore & $\mathrm{R}$ & $\mathrm{FB}$ \\
\hline & Rhipiduridae & & & & & & & \\
\hline 289 & White-browed Fantail & Rhipidura aureola (Lesson, 1830) & कुमथोप्ले मारुनी चरी & LC & - & Insectivore & $\mathrm{R}$ & $\mathrm{FB}$ \\
\hline \multirow[t]{2}{*}{290} & White-throated Fantail & $\begin{array}{l}\text { Rhipidura albicollis (Vieillot, } \\
\text { 1818) }\end{array}$ & नक्कले मारुनी चरी & LC & - & Insectivore & $\mathrm{R}$ & $\mathrm{FB}$ \\
\hline & Scotocercidae & & & & & & & \\
\hline 291 & Chestnut-headed Tesia & $\begin{array}{l}\text { Cettia castaneocoronata (Burton, } \\
\text { 1836) }\end{array}$ & रातो टाउके टेसिया & LC & - & Insectivore & $\mathrm{R}$ & $\mathrm{FB}$ \\
\hline \multirow[t]{2}{*}{292} & $\begin{array}{l}\text { Pale-footed Bush- } \\
\text { warbler }\end{array}$ & $\begin{array}{l}\text { Hemitesia pallidipes* (Blanford, } \\
1872 \text { ) }\end{array}$ & घेघरी भाडीफिस्टो & LC & - & Insectivore & $\mathrm{R}$ & $\mathrm{FB}$ \\
\hline & Sittidae & & & & & & & \\
\hline 293 & $\begin{array}{l}\text { Chestnut-bellied } \\
\text { Nuthatch }\end{array}$ & Sitta cinnamoventris (Blyth, 1842) & कटुसे मट्टा & LC & - & Insectivore & $\mathrm{R}$ & $\mathrm{FB}$ \\
\hline \multirow[t]{2}{*}{294} & Velvet-fronted Nuthatch & Sitta frontalis (Swainson, 1820) & मखमली मट्टा & LC & - & Insectivore & $\mathrm{R}$ & $\mathrm{FB}$ \\
\hline & Stenostiridae & & & & & & & \\
\hline 295 & $\begin{array}{l}\text { Grey-headed Canary- } \\
\text { flycatcher }\end{array}$ & $\begin{array}{l}\text { Culicicapa ceylonensis (Swainson, } \\
\text { 1820) }\end{array}$ & चन्चले अर्जुनक & LC & - & Insectivore & W & $\mathrm{FB}$ \\
\hline \multirow[t]{2}{*}{296} & $\begin{array}{l}\text { Yellow-bellied Fairy- } \\
\text { fantail }\end{array}$ & $\begin{array}{l}\text { Chelidorhynx hypoxanthus } \$ \\
\text { (Blyth, 1843) }\end{array}$ & पहेंलो मारुनीचरी & LC & - & Insectivore & $\mathrm{R}$ & $\mathrm{FB}$ \\
\hline & Sturnidae & & & & & & & \\
\hline 297 & Asian Pied Starling & $\begin{array}{l}\text { Gracupica contra (Linnaeus, } \\
\text { 1758) }\end{array}$ & करचुली मैना & LC & - & Omnivore & $\mathrm{R}$ & OF \\
\hline 298 & Bank Myna & $\begin{array}{l}\text { Acridotheres ginginianus } \\
\text { (Latham, 1790) }\end{array}$ & भित्ते सारौं & LC & - & Omnivore & $\mathrm{R}$ & OF \\
\hline 299 & Brahminy Starling & $\begin{array}{l}\text { Sturnia pagodarum\# (Gmelin, } \\
\text { 1789) }\end{array}$ & जुरे सारौं & LC & - & Omnivore & $\mathrm{R}$ & OF \\
\hline 300 & Chestnut-tailed Starling & $\begin{array}{l}\text { Sturnia malabarica\# (Gmelin, } \\
\text { 1789) }\end{array}$ & बगाले सारौं & LC & - & Omnivore & $\mathrm{R}$ & $\mathrm{FB}$ \\
\hline 301 & Common Hill Myna & Gracula religiosa (Linnaeus, 1758) & मैनाचरी & LC & ॥ & Omnivore & $\mathrm{R}$ & FB \\
\hline 302 & Common Myna & $\begin{array}{l}\text { Acridotheres tristis (Linnaeus, } \\
\text { 1766) }\end{array}$ & डाङग्रे सारौं & LC & - & Omnivore & $\mathrm{R}$ & OF \\
\hline 303 & Jungle Myna & $\begin{array}{l}\text { Acridotheres fuscus (Wagler, } \\
\text { 1827)[ }\end{array}$ & काली सारौं & LC & - & Omnivore & $\mathrm{R}$ & $\mathrm{FB}$ \\
\hline 304 & Spot-winged Starling & $\begin{array}{l}\text { Saroglossa spilopterus (Vigors, } \\
1831 \text { ) }\end{array}$ & कटुसकण्ठे सारौं & LC & - & Omnivore & $\mathrm{R}$ & $\mathrm{FB}$ \\
\hline
\end{tabular}




\begin{tabular}{|c|c|c|c|c|c|c|c|c|}
\hline & $\begin{array}{l}\text { Order/ Family/ Common } \\
\text { name }\end{array}$ & Scientific name & Nepali name & IUCN & CITES & $\begin{array}{l}\text { Foraging } \\
\text { guild }\end{array}$ & $\begin{array}{l}\text { Migration } \\
\text { group }\end{array}$ & $\begin{array}{l}\text { Habitat } \\
\text { Type }\end{array}$ \\
\hline & Sylviidae & & & & & & & \\
\hline \multirow[t]{2}{*}{305} & Yellow-eyed Babbler & $\begin{array}{l}\text { Chrysomma sinense (Gmelin, } \\
\text { 1789) }\end{array}$ & तामे घाँसेभ्याँकुर & LC & - & Insectivore & $\mathrm{R}$ & GB \\
\hline & Timaliidae & & & & & & & \\
\hline 306 & Black-chinned Babbler & $\begin{array}{l}\text { Cyanoderma pyrrhops (Blyth, } \\
\text { 1844) }\end{array}$ & कालो चीउंडे वनभ्याँकुर & LC & - & Insectivore & $\mathrm{R}$ & $\mathrm{FB}$ \\
\hline 307 & $\begin{array}{l}\text { Chestnut-capped } \\
\text { Babbler }\end{array}$ & Timalia pileata (Horsfield, 1821) & रातो टाउके घाँसेभ्याँकुर & LC & - & Insectivore & $\mathrm{R}$ & GB \\
\hline \multirow[t]{2}{*}{308} & Pin-Striped Tit Babbler & $\begin{array}{l}\text { Mixornis gularis\# (Horsfield, } \\
\text { 1822) }\end{array}$ & चर्याचर्यारे फिस्टेभ्याँकुर & LC & - & Insectivore & $\mathrm{R}$ & $\mathrm{FB}$ \\
\hline & Turdidae & & & & & & & \\
\hline 309 & Alpine Thrush & $\begin{array}{l}\text { Zoothera mollissima* (Blyth, } \\
\text { 1842) }\end{array}$ & सादाढाडे चाँचर & LC & - & Insectivore & W & $\mathrm{FB}$ \\
\hline 310 & Black-throated Thrush & Turdus atrogularis (Jarocki, 1819) & बगाले चाचर & LC & - & Insectivore & W & $\mathrm{FB}$ \\
\hline 311 & Grey-winged Blackbird & Turdus boulboul (Latham, 1790) & मदना चाचर & LC & - & Insectivore & $\mathrm{R}$ & $\mathrm{FB}$ \\
\hline 312 & Orange-headed Thrush & Geokichla citrina (Latham, 1790) & सुन्तले चाचर & LC & - & Insectivore & S & $\mathrm{FB}$ \\
\hline 313 & Red-throated Thrush & Turdus ruficollis (Pallas, 1776) & रातो कण्ठे चाचर & LC & - & Omnivore & W & $\mathrm{FB}$ \\
\hline 314 & Scaly Thrush & Zoothera dauma (Latham, 1790) & गोब्रे चाचर & LC & - & Insectivore & R & $\mathrm{FB}$ \\
\hline \multirow[t]{2}{*}{315} & Tickell's Thrush & Turdus unicolor (Tickell, 1833) & फुस्ते चाचर & LC & - & Insectivore & W & $\mathrm{FB}$ \\
\hline & Vangidae & & & & & & & \\
\hline 316 & $\begin{array}{l}\text { Bar-winged Flycatcher- } \\
\text { shrike }\end{array}$ & Hemipus picatus (Sykes, 1832) & आसकोटे & LC & - & Insectivore & $\mathrm{R}$ & FB \\
\hline 317 & Common Woodshrike & $\begin{array}{l}\text { Tephrodornis pondicerianus } \\
\text { (Gmelin, 1789) }\end{array}$ & टेन्था & LC & - & Insectivore & $\mathrm{R}$ & $\mathrm{FB}$ \\
\hline 318 & $\begin{array}{l}\text { Indian Paradise- } \\
\text { flycatcher }\end{array}$ & $\begin{array}{l}\text { Terpsiphone paradisi (Linnaeus, } \\
\text { 1758) }\end{array}$ & श्वर्ग चरी & LC & - & Insectivore & $\mathrm{R}$ & $\mathrm{FB}$ \\
\hline 319 & Large Woodshrike & $\begin{array}{l}\text { Tephrodornis virgatus\# } \\
\text { (Temminck, 1824) }\end{array}$ & ठुलो टेन्था & LC & - & Insectivore & $\mathrm{R}$ & $\mathrm{FB}$ \\
\hline \multirow[t]{2}{*}{320} & Red-billed Blue Magpie & $\begin{array}{l}\text { Urocissa erythroryncha } \\
\text { (Boddaert, 1783) }\end{array}$ & स्यालपोथरी लामपुछे & LC & - & Frugivore & $\mathrm{R}$ & $\mathrm{FB}$ \\
\hline & Vireonidae & & & & & & & \\
\hline \multirow[t]{2}{*}{321} & White-bellied Erpornis & $\begin{array}{l}\text { Erpornis zantholeuca\# (Blyth, } \\
\text { 1844) }\end{array}$ & सेतोपेटे जुरेफिस्टो & LC & - & Omnivore & $\mathrm{R}$ & $\mathrm{FB}$ \\
\hline & Zosteropidae & & & & & & & \\
\hline \multirow[t]{3}{*}{322} & Oriental White-eye & $\begin{array}{l}\text { Zosterops palpebrosus } \\
\text { (Temminck, 1824) }\end{array}$ & कांकीर & LC & - & Insectivore & $\mathrm{R}$ & $\mathrm{FB}$ \\
\hline & PELECANIFORMES & & & & & & & \\
\hline & Ardeidae & & & & & & & \\
\hline 323 & $\begin{array}{l}\text { Black-crowned Night- } \\
\text { heron }\end{array}$ & $\begin{array}{l}\text { Nycticorax nycticorax (Linnaeus, } \\
\text { 1758) }\end{array}$ & बाँके बकुल्ला देउकाग & LC & - & Piscivore & $S$ & WB \\
\hline 324 & Cattle Egret & Bubulcus ibis (Linnaeus, 1758) & बस्तु बकुल्ला & LC & - & Piscivore & $\mathrm{R}$ & WB \\
\hline 325 & Cinnamon Bittern & $\begin{array}{l}\text { Ixobrychus cinnamomeus } \\
\text { (Gmelin, 1789) }\end{array}$ & गेरु बकुल्ला & LC & - & Piscivore & $S$ & WB \\
\hline 326 & Great White Egret & Ardea alba\# (Linnaeus, 1758) & ठूलो सेतो बकुल्ला & LC & - & Piscivore & $\mathrm{R}$ & WB \\
\hline 327 & Green-backed Heron & Butorides striata (Linnaeus, 1758) & छोटोखुट्टे बकुल्ला & LC & - & Piscivore & $\mathrm{R}$ & WB \\
\hline 328 & Grey Heron & Ardea cinerea (Linnaeus, 1758) & फुसे बकुल्ला & LC & - & Piscivore & W & WB \\
\hline 329 & Indian Pond-heron & Ardeola grayii (Sykes, 1832) & भक्ति बकुल्ला & LC & - & Piscivore & $\mathrm{R}$ & WB \\
\hline 330 & Intermediate Egret & Ardea intermedia\# (Wagler, 1827) & मभौला सेतो बकुल्ला & LC & - & Piscivore & $\mathrm{R}$ & WB \\
\hline 331 & Little Egret & Egretta garzetta (Linnaeus, 1766) & सानो सेतो बकुल्ला & LC & - & Piscivore & $\mathrm{R}$ & WB \\
\hline 332 & Purple Heron & Ardea purpurea (Linnaeus, 1766) & प्याजी बकुल्ला & LC & - & Piscivore & $\mathrm{R}$ & WB \\
\hline \multirow[t]{2}{*}{333} & Yellow Bittern & $\begin{array}{l}\text { Ixobrychus sinensis (Gmelin, } \\
\text { 1789) }\end{array}$ & पहेंलो जुन बकुल्ला & LC & - & Carnivore & $S$ & WB \\
\hline & Pelecanidae & & & & & & & \\
\hline 334 & Great White Pelican & $\begin{array}{l}\text { Pelecanus onocrotalus\% } \\
\text { (Linnaeus, 1758) }\end{array}$ & ठूलो घाउँके हावासील & LC & - & Piscivore & $P$ & WB \\
\hline
\end{tabular}




\begin{tabular}{|c|c|c|c|c|c|c|c|c|}
\hline & $\begin{array}{l}\text { Order/ Family/ Common } \\
\text { name }\end{array}$ & Scientific name & Nepali name & IUCN & CITES & $\begin{array}{l}\text { Foraging } \\
\text { guild }\end{array}$ & $\begin{array}{l}\text { Migration } \\
\text { group }\end{array}$ & $\begin{array}{l}\text { Habitat } \\
\text { Type }\end{array}$ \\
\hline & Threskiornithidae & & & & & & & \\
\hline \multirow[t]{3}{*}{335} & Red-naped Ibis & $\begin{array}{l}\text { Pseudibis papillosa (Temminck, } \\
\text { 1824) }\end{array}$ & कर्रा साँवरी & LC & - & $\begin{array}{c}\text { Aquatic } \\
\text { invertebrates }\end{array}$ & $\mathrm{R}$ & WB \\
\hline & PICIFORMES & & & & & & & \\
\hline & Megalaimidae & & & & & & & \\
\hline 336 & Blue-throated Barbet & $\begin{array}{l}\text { Psilopogon asiaticus\# (Latham, } \\
\text { 1790) }\end{array}$ & कुथुर्के & LC & - & Frugivore & $\mathrm{R}$ & $\mathrm{FB}$ \\
\hline 337 & Coppersmith Barbet & $\begin{array}{l}\text { Psilopogon haemacephalus\# } \\
\text { (Statius Müller, 1776) }\end{array}$ & बाँणा चरो ( मिलचरो ) & LC & - & Frugivore & $\mathrm{R}$ & $\mathrm{FB}$ \\
\hline 338 & Great Barbet & $\begin{array}{l}\text { Psilopogon virens\# (Boddaert, } \\
\text { 1783) }\end{array}$ & न्याउली & LC & - & Frugivore & $\mathrm{R}$ & $\mathrm{FB}$ \\
\hline \multirow[t]{2}{*}{339} & Lineated Barbet & $\begin{array}{l}\text { Psilopogon lineatus\# (Vieillot, } \\
\text { 1816) }\end{array}$ & छिर्के कुथुर्के & LC & - & Frugivore & $\mathrm{R}$ & $\mathrm{FB}$ \\
\hline & Picidae & & & & & & & \\
\hline 340 & Black-rumped Flameback & $\begin{array}{l}\text { Dinopium benghalense (Linnaeus, } \\
\text { 1758) }\end{array}$ & कालोढाडे लाहाँचे & LC & - & Insectivore & $\mathrm{R}$ & $\mathrm{FB}$ \\
\hline 341 & Buff-spotted Flameback & $\begin{array}{l}\text { Chrysocolaptes lucidus (Scopoli, } \\
\text { 1786) }\end{array}$ & गर्दनथोप्ले लाहाँचे & LC & - & Insectivore & $\mathrm{R}$ & $\mathrm{FB}$ \\
\hline 342 & Eurasian Wryneck & Jynx torquilla (Linnaeus, 1758) & खर लाहाँचे & LC & - & Insectivore & W & GB \\
\hline 343 & $\begin{array}{l}\text { Fulvous-breasted } \\
\text { Woodpecker }\end{array}$ & $\begin{array}{l}\text { Dendrocopos macei\# (Vieillot, } \\
\text { 1818) }\end{array}$ & काष्ठकुट & LC & - & Insectivore & $\mathrm{R}$ & $\mathrm{FB}$ \\
\hline 344 & Greater Yellownape & $\begin{array}{l}\text { Chrysophlegma flavinucha } \\
\text { (Gould, 1834) }\end{array}$ & ठूलो सुनजुरे काठफोर & LC & - & Insectivore & $\mathrm{R}$ & $\mathrm{FB}$ \\
\hline 345 & $\begin{array}{l}\text { Grey-capped } \\
\text { Woodpecker }\end{array}$ & $\begin{array}{l}\text { Picoides canicapillus\# (Blyth, } \\
\text { 1845) }\end{array}$ & फुस्रे टाउके काष्ठकुट & LC & - & Insectivore & $\mathrm{R}$ & $\mathrm{FB}$ \\
\hline 346 & Grey-faced Woodpecker & Picus canus (Gmelin, 1788) & कालो गर्धने काठफोर & LC & - & Insectivore & $\mathrm{R}$ & $\mathrm{FB}$ \\
\hline 347 & Himalayan Flameback & Dinopium shorii (Vigors, 1832) & तीनऔंले लाहांचे & LC & - & Insectivore & $R$ & $\mathrm{FB}$ \\
\hline 348 & Lesser Yellownape & Picus chlorolophus (Vieillot, 1818) & सुन जुरे काठफोर & LC & - & Insectivore & $\mathrm{R}$ & $\mathrm{FB}$ \\
\hline 349 & Rufous Woodpecker & $\begin{array}{l}\text { Micropternus brachyurus\# } \\
\text { (Vieillot, 1818) }\end{array}$ & सानो तामे काष्ठकुट & LC & - & Insectivore & $\mathrm{R}$ & $\mathrm{FB}$ \\
\hline 350 & $\begin{array}{l}\text { Scaly-bellied } \\
\text { Woodpecker }\end{array}$ & Picus squamatus (Vigors, 1831) & ठूलोकत्ले काठफोर & LC & - & Insectivore & $\mathrm{R}$ & $\mathrm{FB}$ \\
\hline 351 & $\begin{array}{l}\text { Streak-throated } \\
\text { Woodpecker }\end{array}$ & $\begin{array}{l}\text { Picus xanthopygaeus (Gray \& } \\
\text { Gray, 1847) }\end{array}$ & कत्ले काठफोर & LC & - & Insectivore & $\mathrm{R}$ & $\mathrm{FB}$ \\
\hline \multirow[t]{3}{*}{352} & $\begin{array}{l}\text { Yellow-crowned } \\
\text { Woodpecker }\end{array}$ & $\begin{array}{l}\text { Leiopicus mahrattensis@ } \\
\text { (Latham, 1801) }\end{array}$ & पहेंलो(टाउके काष्ठकुट & LC & - & Insectivore & $\mathrm{R}$ & $\mathrm{FB}$ \\
\hline & PODICIPEDIFORMES & & & & & & & \\
\hline & Podicipedidae & & & & & & & \\
\hline 353 & Black-necked Grebe & Podiceps nigricollis (Brehm, 1831) & कालीकण्ठे ढुबुल्के चरा & LC & - & Carnivore & w & WB \\
\hline 354 & Great Crested Grebe & $\begin{array}{l}\text { Podiceps cristatus (Linnaeus, } \\
1758 \text { ) }\end{array}$ & सिउरे ढुबुल्केचरा & LC & - & Piscivore & W & WB \\
\hline \multirow[t]{3}{*}{355} & Little Grebe & $\begin{array}{l}\text { Tachybaptus ruficollis (Pallas, } \\
\text { 1764) }\end{array}$ & ढुबुल्केचरा & LC & - & Carnivore & $\mathrm{R}$ & WB \\
\hline & PSITTACIFORMES & & & & & & & \\
\hline & Psittacidae & & & & & & & \\
\hline 356 & Alexandrine Parakeet & $\begin{array}{l}\text { Psittacula eupatria (Linnaeus, } \\
\text { 1766) }\end{array}$ & कर्रा सुगा & NT & II & Frugivore & $\mathrm{R}$ & $\mathrm{FB}$ \\
\hline 357 & Plum-headed Parakeet & $\begin{array}{l}\text { Psittacula cyanocephala } \\
\text { (Linnaeus, 1766) }\end{array}$ & टुइसी सुगा & LC & II & Frugivore & $\mathrm{R}$ & OF \\
\hline 358 & Red-breasted Parakeet & $\begin{array}{l}\text { Psittacula alexandri (Linnaeus, } \\
\text { 1758) }\end{array}$ & कागभेला सुगा & NT & II & Frugivore & $\mathrm{R}$ & $\mathrm{FB}$ \\
\hline 359 & Rose-ringed Parakeet & Psittacula krameri (Scopoli, 1769) & कण्ठे सुगा & LC & - & Frugivore & $\mathrm{R}$ & $\mathrm{FB}$ \\
\hline \multirow[t]{3}{*}{360} & Slaty-headed Parakeet & $\begin{array}{l}\text { Psittacula himalayana* (Lesson, } \\
\text { 1832) }\end{array}$ & मदना सुगा & LC & II & Frugivore & $\mathrm{R}$ & $\mathrm{FB}$ \\
\hline & STRIGIFORMES & & & & & & & \\
\hline & Strigidae & & & & & & & \\
\hline 361 & Asian Barred Owlet & $\begin{array}{l}\text { Glaucidium cuculoides (Vigors, } \\
\text { 1831) }\end{array}$ & पाते लाटोकोसेरो & LC & II & Carnivore & $\mathrm{R}$ & $\mathrm{FB}$ \\
\hline 362 & Brown Boobook & Ninox scutulata (Raffles, 1822) & हुक्के लाटोकोसेरो & LC & ॥ & Carnivore & R & $\mathrm{FB}$ \\
\hline
\end{tabular}




\begin{tabular}{|c|c|c|c|c|c|c|c|c|}
\hline & $\begin{array}{l}\text { Order/ Family/ Common } \\
\text { name }\end{array}$ & Scientific name & Nepali name & IUCN & CITES & $\begin{array}{l}\text { Foraging } \\
\text { guild }\end{array}$ & $\begin{array}{l}\text { Migration } \\
\text { group }\end{array}$ & $\begin{array}{c}\text { Habitat } \\
\text { Type }\end{array}$ \\
\hline 363 & Brown Fish-owl & $\begin{array}{l}\text { Ketupa zeylonensis (Gmelin, } \\
\text { 1788) }\end{array}$ & हुचील & LC & II & Carnivore & $\mathrm{R}$ & $\mathrm{FB}$ \\
\hline 364 & Collared Owlet & $\begin{array}{l}\text { Glaucidium brodiei* (Burton, } \\
\text { 1836) }\end{array}$ & सानो डुन्डुल & LC & II & Carnivore & W & $\mathrm{FB}$ \\
\hline 365 & Indian Scops-owl & $\begin{array}{l}\text { Otus bakkamoena \# (Pennant, } \\
\text { 1769) }\end{array}$ & चित्री उल्लु & LC & II & Carnivore & $\mathrm{R}$ & $\mathrm{FB}$ \\
\hline 366 & Jungle Owlet & $\begin{array}{l}\text { Glaucidium radiatum (Tickell, } \\
\text { 1833) }\end{array}$ & कुर्कुरे लाटोकोसेरो & LC & II & Carnivore & $\mathrm{R}$ & $\mathrm{FB}$ \\
\hline 367 & Oriental Scops-owl & Otus sunia (Hodgson, 1836) & लोखके उल्लु & LC & ॥ & Carnivore & $\mathrm{R}$ & $\mathrm{FB}$ \\
\hline 368 & Spot-bellied Eagle-owl & Bubo nipalensis (Hodgson, 1836) & कत्ले उल्लु & LC & II & Carnivore & $\mathrm{R}$ & $\mathrm{FB}$ \\
\hline \multirow[t]{3}{*}{369} & Spotted Owlet & Athene brama (Temminck, 1821) & कोचलगाडे लाटोकोसेरो & LC & II & Carnivore & $\mathrm{R}$ & OF \\
\hline & SULIFORMES & & & & & & & \\
\hline & Anhingidae & & & & & & & \\
\hline \multirow[t]{2}{*}{370} & Oriental Darter & $\begin{array}{l}\text { Anhinga melanogaster (Pennant, } \\
\text { 1769) }\end{array}$ & सुइरोठुडे जलचरी & NT & - & Piscivore & $\mathrm{R}$ & WB \\
\hline & Phalacrocoracidae & & & & & & & \\
\hline 371 & Great Cormorant & $\begin{array}{l}\text { Phalacrocorax carbo (Linnaeus, } \\
\text { 1758) }\end{array}$ & जलेवा & LC & - & Piscivore & W & WB \\
\hline 372 & Little Cormorant & Microcarbo niger (Vieillot, 1817) & सानो जलेवा & LC & - & Piscivore & $\mathrm{R}$ & WB \\
\hline
\end{tabular}

W-winter migratory | S-summer migratory | P-passage | R-Resident |*-not recorded in 2015-2016 survey |\#-not recorded in 2002-2012 survey | @ \& \$Adhikari et al. 2003 \& Adhikari 2000 that were not recorded in both surveys | \%-spotted by Bird Education Society. Migration group and foraging guild were taken from IUCN Red List (IUCN 2020) and Indian Biodiversity Portal (2020).

Decline of the North American avifauna. Science 366: 120-124.

Schrauth, F.E. \& M. Wink (2018). Changes in species composition of birds and declining number of breeding territories over 40 years in a nature conservation area in Southwest Germany. Diversity 10: 97. Swan, G.E., R. Cuthbert, M. Quevedo, R.E. Green, D.J. Pain, P. Bartels, A.A. Cunningham, N. Duncan, A.A. Meharg, J.L. Oaks, J. ParryJones, S. Shultz, M.A. Taggart, G. Verdoorn \& K. Wolter (2006).
Toxicity of diclofenac to Gyps vultures. Biology Letters 2(2): 279282. https://doi.org/10.1098/rsbl.2005.0425

Swarup, D., R.C. Patra, V. Prakash, R. Cuthbert, D. Das, P. Avari, D.J. Pain, R.E. Green, A.K. Sharma, M. Saini, D. Das \& M. Taggart (2007). Safety of meloxicam to critically endangered Gyps vultures and other scavenging birds in India. Animal Conservation 10: 192-198.

Thapa, I. (2009). An Overview of Vulture Conservation in Nepal. The Initiation 3: 114-118. 
Dr. Albert G. Orr, Griffith University, Nathan, Australia

Dr. Sameer Padhye, Katholieke Universiteit Leuven, Belgium

Dr. Nancy van der Poorten, Toronto, Canada

Dr. Kareen Schnabel, NIWA, Wellington, New Zealand

Dr. R.M. Sharma, (Retd.) Scientist, Zoological Survey of India, Pune, India

Dr. Manju Siliwal, WILD, Coimbatore, Tamil Nadu, India

Dr. G.P. Sinha, Botanical Survey of India, Allahabad, India

Dr. K.A. Subramanian, Zoological Survey of India, New Alipore, Kolkata, India

Dr. P.M. Sureshan, Zoological Survey of India, Kozhikode, Kerala, India

Dr. R. Varatharajan, Manipur University, Imphal, Manipur, India

Dr. Eduard Vives, Museu de Ciències Naturals de Barcelona, Terrassa, Spain

Dr. James Young, Hong Kong Lepidopterists' Society, Hong Kong

Dr. R. Sundararaj, Institute of Wood Science \& Technology, Bengaluru, India

Dr. M. Nithyanandan, Environmental Department, La Ala Al Kuwait Real Estate. Co. K.S.C., Kuwait

Dr. Himender Bharti, Punjabi University, Punjab, India

Mr. Purnendu Roy, London, UK

Dr. Saito Motoki, The Butterfly Society of Japan, Tokyo, Japan

Dr. Sanjay Sondhi, TITLI TRUST, Kalpavriksh, Dehradun, India

Dr. Nguyen Thi Phuong Lien, Vietnam Academy of Science and Technology, Hanoi, Vietnam

Dr. Nitin Kulkarni, Tropical Research Institute, Jabalpur, India

Dr. Robin Wen Jiang Ngiam, National Parks Board, Singapore

Dr. Lional Monod, Natural History Museum of Geneva, Genève, Switzerland.

Dr. Asheesh Shivam, Nehru Gram Bharti University, Allahabad, India

Dr. Rosana Moreira da Rocha, Universidade Federal do Paraná, Curitiba, Brasil

Dr. Kurt R. Arnold, North Dakota State University, Saxony, Germany

Dr. James M. Carpenter, American Museum of Natural History, New York, USA

Dr. David M. Claborn, Missouri State University, Springfield, USA

Dr. Kareen Schnabel, Marine Biologist, Wellington, New Zealand

Dr. Amazonas Chagas Júnior, Universidade Federal de Mato Grosso, Cuiabá, Brasil

Mr. Monsoon Jyoti Gogoi, Assam University, Silchar, Assam, India

Dr. Heo Chong Chin, Universiti Teknologi MARA (UiTM), Selangor, Malaysia

Dr. R.J. Shiel, University of Adelaide, SA 5005, Australia

Dr. Siddharth Kulkarni, The George Washington University, Washington, USA

Dr. Priyadarsanan Dharma Rajan, ATREE, Bengaluru, India

Dr. Phil Alderslade, CSIRO Marine And Atmospheric Research, Hobart, Australia

Dr. John E.N. Veron, Coral Reef Research, Townsville, Australia

Dr. Daniel Whitmore, State Museum of Natural History Stuttgart, Rosenstein, Germany.

Dr. Yu-Feng Hsu, National Taiwan Normal University, Taipei City, Taiwan

Dr. Keith V. Wolfe, Antioch, California, USA

Dr. Siddharth Kulkarni, The Hormiga Lab, The George Washington University, Washington,

D.C., USA

Dr. Tomas Ditrich, Faculty of Education, University of South Bohemia in Ceske

Budejovice, Czech Republic

Dr. Mihaly Foldvari, Natural History Museum, University of Oslo, Norway

Dr. V.P. Uniyal, Wildlife Institute of India, Dehradun, Uttarakhand 248001, India

Dr. John T.D. Caleb, Zoological Survey of India, Kolkata, West Bengal, India

Dr. Priyadarsanan Dharma Rajan, Ashoka Trust for Research in Ecology and the Environment (ATREE), Royal Enclave, Bangalore, Karnataka, India

\section{Fishes}

Dr. Neelesh Dahanukar, IISER, Pune, Maharashtra, India

Dr. Topiltzin Contreras MacBeath, Universidad Autónoma del estado de Morelos, México

Dr. Heok Hee Ng, National University of Singapore, Science Drive, Singapore

Dr. Rajeev Raghavan, St. Albert's College, Kochi, Kerala, India

Dr. Robert D. Sluka, Chiltern Gateway Project, A Rocha UK, Southall, Middlesex, UK

Dr. E. Vivekanandan, Central Marine Fisheries Research Institute, Chennai, India

Dr. Davor Zanella, University of Zagreb, Zagreb, Croatia

Dr. A. Biju Kumar, University of Kerala, Thiruvananthapuram, Kerala, India

Dr. Akhilesh K.V., ICAR-Central Marine Fisheries Research Institute, Mumbai Research

Centre, Mumbai, Maharashtra, India

Dr. J.A. Johnson, Wildlife Institute of India, Dehradun, Uttarakhand, India

\section{Amphibians}

Dr. Sushil K. Dutta, Indian Institute of Science, Bengaluru, Karnataka, India

Dr. Annemarie Ohler, Muséum national d'Histoire naturelle, Paris, France

\section{Reptiles}

Dr. Gernot Vogel, Heidelberg, Germany

Dr. Raju Vyas, Vadodara, Gujarat, India

Dr. Pritpal S. Soorae, Environment Agency, Abu Dubai, UAE.

Prof. Dr. Wayne J. Fuller, Near East University, Mersin, Turkey

Prof. Chandrashekher U. Rivonker, Goa University, Taleigao Plateau, Goa. India

Dr. S.R. Ganesh, Chennai Snake Park, Chennai, Tamil Nadu, India

Dr. Himansu Sekhar Das, Terrestrial \& Marine Biodiversity, Abu Dhabi, UAE
Birds

Dr. Hem Sagar Baral, Charles Sturt University, NSW Australia

Dr. Chris Bowden, Royal Society for the Protection of Birds, Sandy, UK

Dr. Priya Davidar, Pondicherry University, Kalapet, Puducherry, India

Dr. J.W. Duckworth, IUCN SSC, Bath, UK

Dr. Rajah Jayapal, SACON, Coimbatore, Tamil Nadu, India

Dr. Rajiv S. Kalsi, M.L.N. College, Yamuna Nagar, Haryana, India

Dr. V. Santharam, Rishi Valley Education Centre, Chittoor Dt., Andhra Pradesh, India

Dr. S. Balachandran, Bombay Natural History Society, Mumbai, India

Mr. J. Praveen, Bengaluru, India

Dr. C. Srinivasulu, Osmania University, Hyderabad, India

Dr. K.S. Gopi Sundar, International Crane Foundation, Baraboo, USA

Dr. Gombobaatar Sundev, Professor of Ornithology, Ulaanbaatar, Mongolia

Prof. Reuven Yosef, International Birding \& Research Centre, Eilat, Israel

Dr. Taej Mundkur, Wetlands International, Wageningen, The Netherlands

Dr. Carol Inskipp, Bishop Auckland Co., Durham, UK

Dr. Tim Inskipp, Bishop Auckland Co, Durham, UK

Dr. V. Gokula, National College, Tiruchirappalli, Tamil Nadu, India

Dr. Arkady Lelej, Russian Academy of Sciences, Vladivostok, Russia

Dr. Simon Dowell, Science Director, Chester Zoo, UK

Dr. Mário Gabriel Santiago dos Santos, Universidade de Trás-os-Montes e Alto Douro,

Quinta de Prados, Vila Real, Portugal

Dr. Grant Connette, Smithsonian Institution, Royal, VA, USA

Dr. M. Zafar-ul Islam, Prince Saud Al Faisal Wildlife Research Center, Taif, Saudi Arabia

Mammals

Dr. Giovanni Amori, CNR - Institute of Ecosystem Studies, Rome, Italy

Dr. Anwaruddin Chowdhury, Guwahati, India

Dr. David Mallon, Zoological Society of London, UK

Dr. Shomita Mukherjee, SACON, Coimbatore, Tamil Nadu, India

Dr. Angie Appel, Wild Cat Network, Germany

Dr. P.O. Nameer, Kerala Agricultural University, Thrissur, Kerala, India

Dr. Ian Redmond, UNEP Convention on Migratory Species, Lansdown, UK

Dr. Heidi S. Riddle, Riddle's Elephant and Wildlife Sanctuary, Arkansas, USA

Dr. Karin Schwartz, George Mason University, Fairfax, Virginia.

Dr. Lala A.K. Singh, Bhubaneswar, Orissa, India

Dr. Mewa Singh, Mysore University, Mysore, India

Dr. Paul Racey, University of Exeter, Devon, UK

Dr. Honnavalli N. Kumara, SACON, Anaikatty P.O., Coimbatore, Tamil Nadu, India

Dr. Nishith Dharaiya, HNG University, Patan, Gujarat, India

Dr. Spartaco Gippoliti, Socio Onorario Società Italiana per la Storia della Fauna "Giuseppe

Altobello", Rome, Italy

Dr. Justus Joshua, Green Future Foundation, Tiruchirapalli, Tamil Nadu, India

Dr. H. Raghuram, The American College, Madurai, Tamil Nadu, India

Dr. Paul Bates, Harison Institute, Kent, UK

Dr. Jim Sanderson, Small Wild Cat Conservation Foundation, Hartford, USA

Dr. Dan Challender, University of Kent, Canterbury, UK

Dr. David Mallon, Manchester Metropolitan University, Derbyshire, UK

Dr. Brian L. Cypher, California State University-Stanislaus, Bakersfield, CA

Dr. S.S. Talmale, Zoological Survey of India, Pune, Maharashtra, India

Prof. Karan Bahadur Shah, Budhanilakantha Municipality, Kathmandu, Nepal

Dr. Susan Cheyne, Borneo Nature Foundation International, Palangkaraja, Indonesia

Dr. Hemanta Kafley, Wildlife Sciences, Tarleton State University, Texas, USA

\section{Other Disciplines}

Dr. Aniruddha Belsare, Columbia MO 65203, USA (Veterinary)

Dr. Mandar S. Paingankar, University of Pune, Pune, Maharashtra, India (Molecular)

Dr. Jack Tordoff, Critical Ecosystem Partnership Fund, Arlington, USA (Communities)

Dr. Ulrike Streicher, University of Oregon, Eugene, USA (Veterinary)

Dr. Hari Balasubramanian, EcoAdvisors, Nova Scotia, Canada (Communities)

Dr. Rayanna Hellem Santos Bezerra, Universidade Federal de Sergipe, São Cristóvão, Brazil

Dr. Jamie R. Wood, Landcare Research, Canterbury, New Zealand

Dr. Wendy Collinson-Jonker, Endangered Wildlife Trust, Gauteng, South Africa

Dr. Rajeshkumar G. Jani, Anand Agricultural University, Anand, Gujarat, India

Dr. O.N. Tiwari, Senior Scientist, ICAR-Indian Agricultural Research Institute (IARI), New

Delhi, India

Dr. L.D. Singla, Guru Angad Dev Veterinary and Animal Sciences University, Ludhiana, India

Dr. Rupika S. Rajakaruna, University of Peradeniya, Peradeniya, Sri Lanka

Dr. Bahar Baviskar, Wild-CER, Nagpur, Maharashtra 440013, India

Reviewers 2018-2020

Due to pausity of space, the list of reviewers for 2018-2020 is available online.
The opinions expressed by the authors do not reflect the views of the Journal of Threatened Taxa, Wildlife Information Liaison Development Society, Zoo Outreach Organization, or any of the partners. The journal, the publisher, the host, and the partners are not responsible for the accuracy of the political boundaries shown in the maps by the authors.

\footnotetext{
Print copies of the Journal are available at cost. Write to:

The Managing Editor, JoTT,

c/o Wildlife Information Liaison Development Society,

No. 12, Thiruvannamalai Nagar, Saravanampatti - Kalapatti Road,

Saravanampatti, Coimbatore, Tamil Nadu 641035, India

ravi@threatenedtaxa.org
} 


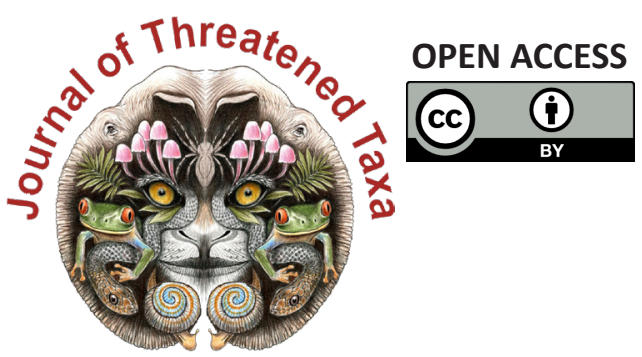

www.threatenedtaxa.org

The Journal of Threatened Taxa (JoTT) is dedicated to building evidence for conservation globally by publishing peer-reviewed articles online every month at a reasonably rapid rate at www.threatenedtaxa.org. All articles published in JoTT are registered under Creative Commons Attribution 4.0 International License unless otherwise mentioned. JoTT allows allows unrestricted use, reproduction, and distribution of articles in any medium by providing adequate credit to the author(s) and the source of publication.

\section{ISSN $0974-7907$ (Online) | ISSN $0974-7893$ (Print)}

\section{September 2021 | Vol. 13 | No. 11 | Pages: 19431-19674 Date of Publication: 26 September 2021 (Online \& Print) DOI: 10.11609/jott.2021.13.11.19431-19674}

Articles

Understanding human-flying fox interactions in the Agusan Marsh Wildlife Sanctuary as basis for conservation policy interventions

- Sherryl L. Paz \& Juan Carlos T. Gonzalez, Pp. 19431-19447

Argentinian odonates (dragonflies and damselflies): current and future distribution and discussion of their conservation

- A. Nava-Bolaños, D.E. Vrech, A.V. Peretti \& A. Córdoba-Aguilar, Pp. 19448-19465

\section{Communications}

The diel activity pattern of small carnivores of Western Ghats, India: a case study at Nelliampathies in Kerala, India

- Devika Sanghamithra \& P.O. Nameer, Pp. 19466-19474

Distribution and threats to Smooth-Coated Otters Lutrogale perspicillata (Mammalia: Carnivora: Mustelidae) in Shuklaphanta National Park, Nepal

- Gopi Krishna Joshi, Rajeev Joshi \& Bishow Poudel, Pp. 19475-19483

Wildlife hunting practices of the Santal and Oraon communities in Rajshahi, Bangladesh - Azizul Islam Barkat, Fahmida Tasnim Liza, Sumaiya Akter, Ashikur Rahman Shome \& M. Fazle Rabbe, Pp. 19484-19491

Ethnozoological use of primates in northeastern India

- Deborah Daolagupu, Nazimur Rahman Talukdar \& Parthankar Choudhury, Pp. 19492-19499

Factors influencing the flush response and flight initiation distance of three owl species in the Andaman Islands

- Shanmugavel Sureshmarimuthu, Santhanakrishnan Babu, Honnavalli Nagaraj Kumara \& Nagaraj Rajeshkumar, Pp. 19500-19508

Birds of Barandabhar Corridor Forest, Chitwan, Nepal

- Saneer Lamichhane, Babu Ram Lamichhane, Kapil Pokharel, Pramod Raj Regmi, Tulasi Prasad Dahal, Santosh Bhattarai, Chiranjibi Prasad Pokheral, Pabitra Gotame,

Trishna Rayamajhi, Ram Chandra Kandel \& Aashish Gurung, Pp. 19509-19526

On some additions to the amphibians of Gunung Inas Forest Reserve, Kedah,

Peninsular Malaysia

- Shahriza Shahrudin, Pp. 19527-19539

Reviews

A review of research on the distribution, ecology, behaviour, and conservation of the Slender Loris Loris lydekkerianus (Mammalia: Primates: Lorisidae) in India

- Mewa Singh, Mridula Singh, Honnavalli N. Kumara, Shanthala Kumar, Smitha D. Gnanaolivu \& Ramamoorthy Sasi, Pp. 19540-19552

Bivalves (Mollusca: Bivalvia) in Malaysian Borneo: status and threats

- Abdulla-Al-Asif, Hadi Hamli, Abu Hena Mustafa Kamal, Mohd Hanafi Idris, Geoffery James Gerusu, Johan Ismail \& Muyassar H. Abualreesh, Pp. 19553-19565

Disentangling earthworm taxonomic stumbling blocks using molecular markers

- Azhar Rashid Lone, Samrendra Singh Thakur, Nalini Tiwari, Olusola B. Sokefun \&

Shweta Yadav, Pp. 19566-19579

A reference of identification keys to plant-parasitic nematodes (Nematoda: Tylenchida) Tylenchomorpha)

- Reza Ghaderi, Manouchehr Hosseinvand \& Ali Eskandari, Pp. 19580-19602

Short Communications

Catalogue of herpetological specimens from Meghalaya, India at the Salim Ali Centre for Ornithology and Natural History

-S.R. Chandramouli, R.S. Naveen, S. Sureshmarimuthu, S. Babu, P.V. Karunakaran \&

Honnavalli N. Kumara, Pp. 19603-19610
A preliminary assessment of odonate diversity along the river Tirthan, Great Himalayan National Park Conservation Area, India with reference to the impact of climate change - Amar Paul Singh, Kritish De, Virendra Prasad Uniyal \& Sambandam Sathyakumar, Pp. 19611-19615

A checklist of orthopteran fauna (Insecta: Orthoptera) with some new records in the cold arid region of Ladakh, India

- M. Ali, M. Kamil Usmani, Hira Naz, Tajamul Hassan Baba \& Mohsin Ali, Pp. 19616-19625

New distribution records of two Begonias to the flora of Bhutan

- Phub Gyeltshen \& Sherab Jamtsho, Pp. 19626-19631

Rediscovery of Aponogeton lakhonensis A. Camus (Aponogetonaceae): a long-lost aquatic plant of India

- Debolina Dey, Shrirang Ramchandra Yadav \& Nilakshee Devi, Pp. 19632-19635

Glyphochloa acuminata (Hack.) Clayton var. laevis (Poaceae): a new variety from central Western Ghats of Karnataka, India

- H.U. Abhijit \& Y.L. Krishnamurthy, Pp. 19636-19639

A cytomorphological investigation of three species of the genus Sonchus L. (Asterales: Asteraceae) from Punjab, India

- M.C. Sidhu \& Rai Singh, Pp. 19640-19644

Dryopteris lunanensis (Dryopteridaceae) - an addition to the pteridophytic diversity of India

- Chhandam Chanda, Christopher Roy Fraser-Jenkins \& Vineet Kumar Rawat, Pp. 1964519648

Notes

First record of Spotted Linsang Prionodon pardicolor (Mammalia: Carnivora:

Prionodontidae) with photographic evidence in Meghalaya, India

- Papori Khatonier \& Adrian Wansaindor Lyngdoh, Pp. 19649-19651

First record of the Eastern Cat Snake Boiga gocool (Gray, 1835) (Squamata: Colubridae) from Tripura, India

- Sumit Nath, Biswajit Singh, Chiranjib Debnath \& Joydeb Majumder, Pp. 19652-19656

First record of the genus Tibetanja (Lepidoptera: Eupterotidae: Janinae) from India - Alka Vaidya \& H. Sankararaman, Pp. 19657-19659

Austroborus cordillerae (Mollusca: Gastropoda) from central Argentina: a rare, little-known land snail

- Sandra Gordillo, Pp. 19660-19662

Intestinal coccidiosis (Apicomplexa: Eimeriidae) in a Himalayan Griffon Vulture Gyps himalayensis

- Vimalraj Padayatchiar Govindan, Parag Madhukar Dhakate \& Ayush Uniyal, Pp. 1966319664

Two new additions to the orchid flora of Assam, India

- Sanswrang Basumatary, Sanjib Baruah \& Lal Ji Singh, Pp. 19665-19670

Wildlife art and illustration - combining black and white ink drawings with colour: some experiments in Auroville, India

- M. Eric Ramanujam \& Joss Brooks, Pp. 19671-19674
Publisher \& Host

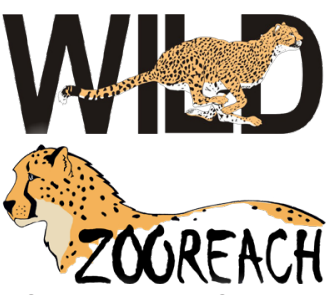

